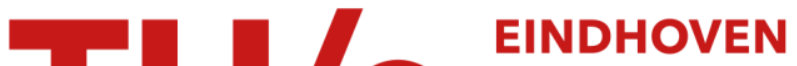 UNIVERSITY OF TECHNOLOGY
}

\section{Code division-based sensing of illumination contributions in solid-state lighting systems}

\section{Citation for published version (APA):}

Linnartz, J. P. M. G., Feri, L., Yang, H., Colak, S. B., \& Schenk, T. C. W. (2009). Code division-based sensing of illumination contributions in solid-state lighting systems. IEEE Transactions on Signal Processing, 57(10), 39843998. https://doi.org/10.1109/TSP.2009.2025075

DOI:

10.1109/TSP.2009.2025075

Document status and date:

Published: 01/01/2009

\section{Document Version:}

Publisher's PDF, also known as Version of Record (includes final page, issue and volume numbers)

\section{Please check the document version of this publication:}

- A submitted manuscript is the version of the article upon submission and before peer-review. There can be important differences between the submitted version and the official published version of record. People interested in the research are advised to contact the author for the final version of the publication, or visit the $\mathrm{DOI}$ to the publisher's website.

- The final author version and the galley proof are versions of the publication after peer review.

- The final published version features the final layout of the paper including the volume, issue and page numbers.

Link to publication

\section{General rights}

Copyright and moral rights for the publications made accessible in the public portal are retained by the authors and/or other copyright owners and it is a condition of accessing publications that users recognise and abide by the legal requirements associated with these rights.

- Users may download and print one copy of any publication from the public portal for the purpose of private study or research.

- You may not further distribute the material or use it for any profit-making activity or commercial gain

- You may freely distribute the URL identifying the publication in the public portal.

If the publication is distributed under the terms of Article 25fa of the Dutch Copyright Act, indicated by the "Taverne" license above, please follow below link for the End User Agreement:

www.tue.nl/taverne

Take down policy

If you believe that this document breaches copyright please contact us at:

openaccess@tue.nl

providing details and we will investigate your claim. 


\title{
Code Division-Based Sensing of Illumination Contributions in Solid-State Lighting Systems
}

\author{
Jean-Paul M. G. Linnartz, Senior Member, IEEE, Lorenzo Feri, Member, IEEE, \\ Hongming Yang, Student Member, IEEE, Sel B. Colak, and Tim C. W. Schenk, Member, IEEE
}

\begin{abstract}
Light-emitting diodes (LEDs) have emerged as a prime candidate for the light source of the future. To enable easy user interaction with a future lighting system consisting of many LEDs, this paper proposes a method to accurately measure and estimate the local light contributions of a large set of LEDs. This is enabled through tagging the light of each LED with an unique identifier. To this end, we propose a new family of modulation and multiple access schemes in this paper, named code-time division multiple access-pulse position modulation (CTDMA-PPM) and CTDMA-pulse width modulation (CTDMA-PWM). These schemes satisfy illumination constraints, are compatible with the commonly used PWM dimming of LEDs, and meet the multi-signal separation requirements for simultaneous measurement of illumination strengths. Based on these modulation methods, the paper develops algorithms to estimate illumination. Finally, performance analyses show that even for a very large number of LEDs, the sensing performance of the proposed system satisfies the requirements up to an adequate range.
\end{abstract}

Index Terms-Code division multiple access, light-emitting diodes, lighting control, pulse position modulation, pulse width modulation.

\section{INTRODUCTION}

$\mathbf{L}$ IGHT-EMITTING diodes (LEDs) will capture a significant portion of the lighting market and may largely replace incandescent and gas discharge lamps in the future [2]. The progress in luminance delivered by LEDs follows an exponential trend of doubling every 18-24 months. By the time of publishing this paper, presumably commercial LEDs will be capable of delivering more than one hundred lumen per device [3]. Various solutions exist to create white light with LEDs, however, luminaires that involve red, green, blue plus possibly intermediate colors give the best perceived color quality of the illumination [4]. Advantages of solid-state lighting (SSL) include the possibility to create light sources in artistic shapes and sizes, or to combine multiple small light sources into one light source.

Manuscript received June 12, 2008; accepted April 26, 2009. First published June 10, 2009; current version published September 16, 2009. The associate editor coordinating the review of this manuscript and approving it for publication was Dr. Peter J. Schreier. Part of this work was presented at the International Conference on Communications, Beijing, China, May 2008 [1].

J.-P. M. G. Linnartz, and H. Yang are with the Philips Research Eindhoven, 5656 AE Eindhoven, The Netherlands. They are also with the Department of Electrical Engineering, Eindhoven University of Technology, 5600 MB Eindhoven, The Netherlands (e-mail: J.P. Linnartz@ philips.com; h.m.yang@tue.nl).

L. Feri, S. B. Colak, and T. C. W. Schenk are with Philips Research Eindhoven, 5656 AE Eindhoven, The Netherlands (e-mail: lorenzo.feri@philips. com; s.b.colak@philips.com; tim.schenk@philips.com).

Color versions of one or more of the figures in this paper are available online at http://ieeexplore.ieee.org.

Digital Object Identifier 10.1109/TSP.2009.2025075
Moreover, combinations of many small LEDs maintain good energy efficiency. In order to deliver different illumination levels, LEDs are typically driven with repeatedly transmitted pulses whose widths are modulated [5], so-called pulse width modulation (PWM).

Control of traditional light sources is mostly limited to intensity control, but does not allow color control. LED technology has the major advantage of additionally allowing the control over the light color and illumination pattern. Hence, the concept of pure illumination may shift into dynamic lighting atmosphere provisioning. Smart and intuitive control of a lighting system containing many LEDs, and consequently many parameters to control, becomes important. Fig. 1 depicts an intelligent lighting control system that enables such intuitive control. The technology presented in this paper is believed to enable new easy-to-use concepts to control an LED lighting environment. This technology is based on uniquely tagging the light emitted by different LEDs through modulation (via "light link c" in Fig. 1), which we will refer to as coded light. Moreover, it includes a sensor receiving these tags and estimating the light intensity of each LED. In an intelligent lighting system, these estimates would be, together with user input on required lighting pattern, transmitted to a master controller (via "control link a") connected to all the LED lamps (via "control link b").

Modulation of LEDs has been studied in several previous works, though initially mainly for infrared (IR) wireless communications [6]-[9]. Recently, code division multiple access (CDMA) for multiuser IR communications has been treated in [10], [11]. Currently one can observe an increased interest in visible light communications using powerful lighting LEDs [12]-[17]. The main focus of these papers is on achieving high-speed data communications with arrays of visible light sources. To this end, techniques incompatible with the commonly applied PWM dimming technique are being proposed, such as orthogonal frequency division multiplexing [14] and discrete multitone [15], or methods requiring high PWM frequencies as in [17]. Moreover, these papers do not apply transmitter-based multiple-access techniques, since most of them consider a common data signal on all LEDs in a system/array, or apply a complicated optical sensor array for signal separation [16]. Consequently, these techniques are not directly applicable for the separate sensing of the illumination contribution of each LED, as considered in this paper. The main performance indicators of our control of the system are, as further detailed in Section II-C, that all light sources can be distinguished individually within the aggregate illumination, that their individual contributions can be estimated regularly, accurately and simultaneously, and that the driving technique of the LEDs is compatible with PWM dimming. However, in 


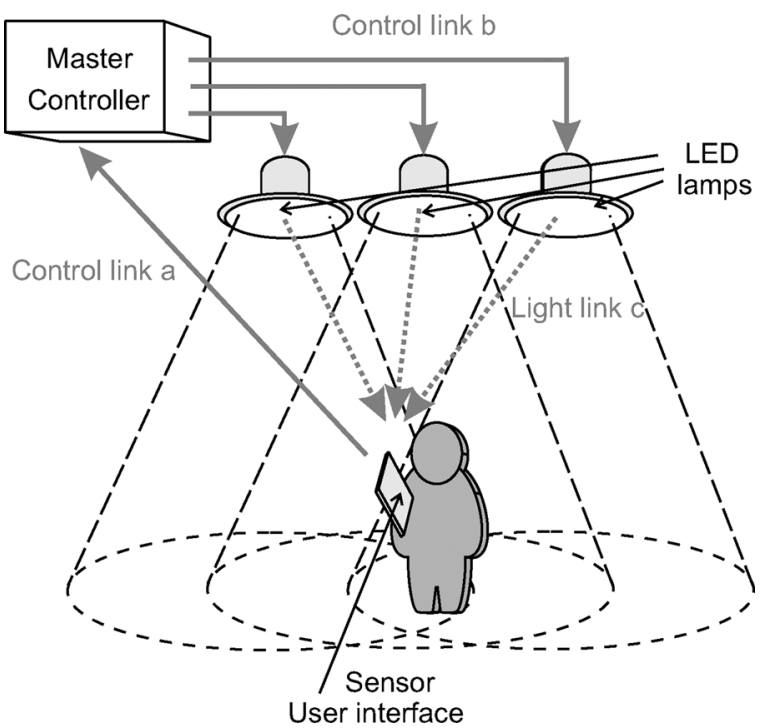

Fig. 1. An illustration of an intelligent lighting control system using coded light.

our case, aggregate data throughput and bit-error rate (BER) are of little or no relevance.

Motivated by the above, we propose two modulation and multiple-access schemes, which combine PWM and pulse position modulation (PPM) of the LED driving waveform with a hybrid code-time division multiple access (CTDMA), along with their multi-signal receiver processing algorithms.

The main contributions of this paper include the following:

- the formulation of lighting control via coded light as a research problem, and the formulation of the associated requirements;

- the proposal of a system concept to enable localized lighting control;

- the proposal of two modulation and multiple access schemes, i.e., CTDMA-PPM and CTDMA-PWM, and the corresponding multi-signal receiver processing, that enable the proposed system concept;

- a theoretical and numerical performance analysis of the proposed system, not only in terms of real-time measurements of the lighting channel propagation properties, but also in terms of perceivable color error in illumination rendering.

This paper is organized as follows. First, Section II introduces the interaction concept, system architecture, and corresponding system requirements considered in this paper. Section III, subsequently, formulates the coded light encoding system and Section IV describes the channel model. In Section V, a receiver structure is introduced and its red-green-blue illumination contribution estimation performance is studied analytically. Section VI, subsequently, studies the influence of imperfect timing on this performance. In Section VII, this is extended towards the accuracy in color point estimation, which is relevant to quantify the user experience of our control system. Finally, Section VIII concludes the paper.

\section{INTELLIGENT Lighting CONTROL SYSTEM}

In this section, we introduce the basics of an intelligent lighting control system. We consider an interaction concept, present the lighting control system architecture and detail the system requirements for the modulation and multiple access scheme.

\section{A. Interaction Concept}

A new interaction model for lighting systems is that the user holds a remote control at the location where he wants to control the illumination settings. He presses the control buttons to set the light effect at that particular location. The lighting system then automatically figures out the light contribution of each light source that is responsible for the illumination at that location and modifies the light settings of the appropriate light sources to achieve the desired effect. To this end, this paper proposes a modulation method in which the light emitted by each light source carries a unique identifying code, which is invisible to the human eye. A sensor in the remote control detects these identifiers, identifies the light sources involved, and measures the strength of the contribution of each LED at the sensor location. This allows the remote control to send a control message to modify the relevant light settings. Moreover, it can measure the resulting changes in a closed control loop.

Our paper contributes to the realization of this concept by proposing a practical, convenient, and efficient approach for embedding an electronically detectable identification code into each light contribution.

\section{B. Control System Architecture}

Fig. 1 schematically describes the considered lighting control system. A control loop is formed by a unidirectional lightwave link ("light link c" in Fig. 1) from one or multiple light sources to a sensor, e.g. placed in a remote control. This link is used to transmit light source identifiers, which allow the sensor to estimate the local illumination contributions of each of the LEDs. The lightwave link is followed by a link from the sensor to a room controller ("control link a" in Fig. 1), and another link from the room controller to the light sources ("control link b" in Fig. 1). The focus of this paper is on the first (multiple-access) link, which coincides with the illumination propagation path. The second link can for instance be a ZigBee radio link, or an infrared (IR) link. The third link can be a radio (broadcast) network, a ZigBee network, a wired Digital Addressable Lighting Interface (DALI), Digital Multiplexing (DMX) network, or involve power line communications. Since the second and third link can be bidirectional, status information from the light sources such as their temperature, and the associated color spectrum of the emitted light can be shared within the control loop. Alternatively, this information could also be transmitted over the light link, see [1]. In this paper, however, we only focus on the use of the light link to enable the estimation of the local illumination contributions.

\section{System Requirements}

We now give a brief account of the requirements posed on the modulation and multiple access schemes for the light link and the corresponding estimation algorithms, such that these are compatible with the commonly used PWM method of controlling the intensity and color point of LED lighting. In this PWM method the LEDs are rapidly switched on and off with an appropriate duty cycle. A lower on-period results in a lower 


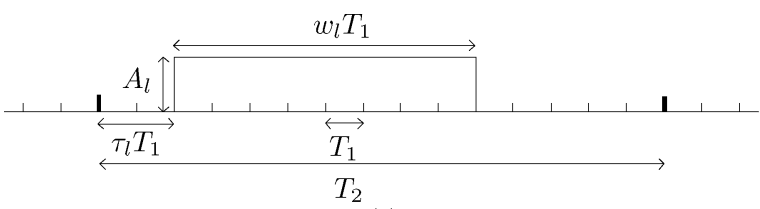

(a)

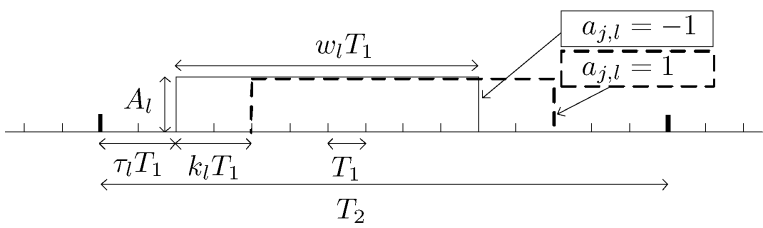

(b)

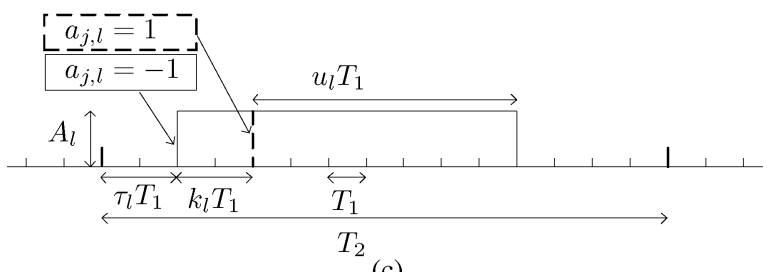

(c)

Fig. 2. Description of the binary waveform pulse formats for the different modulation schemes. (a) Non-modulated pulse. (b) Pulse position modulation. (c) Pulse width modulation.

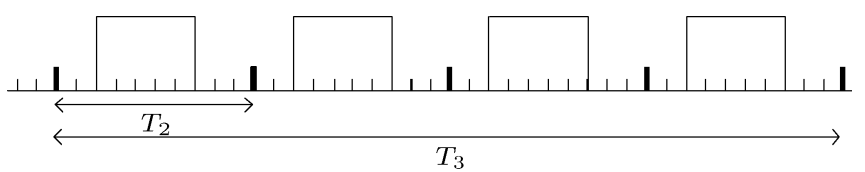

(a)

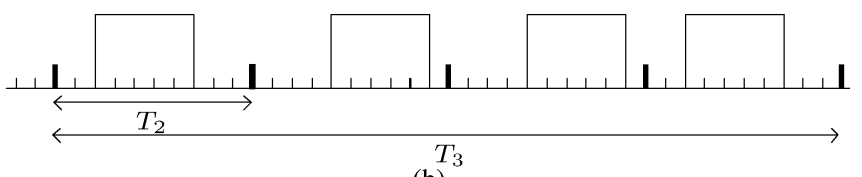

(b)

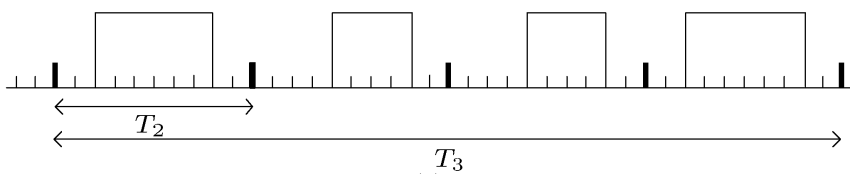

(c)

Fig. 3. Example of the pulse train for one frame for an LED. Code $\mathbf{a}=[-1,1,1,-1], p_{l}=0.5, k_{l}=2$, and $\tau_{l}=2$. (a) Non-modulated. (b) CTDMA-PPM. (c) CTDMA-PWM.

average light output level of the LED and, hence, corresponds to a higher dimming. This dimming concept is illustrated by the LED driving waveforms depicted in Figs. 2(a) and 3(a), which are a single PWM pulse and sequence of PWM pulses, respectively. Changes in color can be achieved by independently changing the dimming levels of colocated LEDs with different colors.

The requirements are listed in order of importance.

a) Independent illumination and light source identification: Since the main application of the considered system is illumination, the modulation scheme should not affect the short time-average duty cycle of any LED. The dimming level has to be treated as an external parameter, which needs to be varied per light source in accordance with the illumination specification to render the desired lighting atmosphere. This may include slow time variations for the generation of dynamic lighting effects and independent dimming level settings from one LED to another.

b) Sufficient dimming resolution: To enable accurate atmosphere and light color rendering, the modulation method should allow a dimming resolution of 8-12 bits, or similarly a contrast ratio of 1:256 to $1: 4096$.

c) Imperceivable modulation: The modulation scheme and the light dimming method should not cause visible flickering. We believe that, due to the large illuminated area, the requirements are more stringent than for displays, where refresh rates of 50 or $100 \mathrm{~Hz}$ are acceptable. In some situations, particularly during rapid eye movements, a human eye can notice frequency components as high as hundreds of Hertz in the illumination [18]. This can be avoided by elimination of low frequency components in the modulation.

d) Accurate estimation: The system has to allow measurements of the illumination intensity and color point of the light at distances where the light contributes to the illumination, distinguishing just noticeable differences defined in human perception models [19], [20].

e) Simultaneous estimation: The system has to be able to measure the contribution from each locally relevant light source individually and simultaneously. It has to operate in an environment with several thousands of LEDs, and be robust against mutual interference.

f) Low complexity: The detector/estimator should be simple and low power, to be suited for use in a remote control device.

g) Interoperability: The modulation scheme has to be usable either with a multicolor light sensor or just with a monochromatic sensor. Moreover, groups of LEDs, e.g., belonging to the same luminaire, may carry the same identifier, or one may assign a separate identifier for each LED (color component).

h) Energy efficiency: The tolerable frequency of switching on-off the power of any LED is limited. Power switching causes capacitive losses in the device and its driver circuitry. To maintain a high power efficiency of the light source, the number of pulses per unit of time needs to be low, say below $1 \mathrm{kHz}$. One can nonetheless consider to use a high clock frequency for a fine resolution in the pulse position, if the illumination pulses are designed to cover a large number of clock cycles without transitions. Moreover, too frequent switching, e.g. if the transients cover more than a few percent of a typical pulse duration, can cause color shifting. During transients, the LED junction is powered differently and typically emits a different spectrum [21], which may create undesirable color artifacts.

i) Robust modulation and estimation: Ambient light, including sunlight, should not harmfully impair accurate estimation of the illumination contributions. The requirements due to shot noise are, however, less stringent than for an optical communication system experiencing ambient light. Since in the considered system, an LED light source needs only to be accurately measurable if its light output power contributes significantly to the local perceived illumination. 
j) Rapid estimation: The modulation method should allow fast estimation. This guarantees that the user experiences an immediate reaction after pressing a control button. Hence, a sensor should be able to identify and measure all relevant light sources within a few $100 \mathrm{~ms}$.

In the remainder of this paper, we will challenge our choices for the modulation system and its parameters against these requirements, and in particular quantify the performance with regard to requirement d).

\section{Modulation AND MultiPle ACCESS}

In this section, we propose the CTDMA-PPM and CTDMA-PWM visible light modulation schemes, which are designed to embed identifiers in the light, while meeting the requirements posed in Section II-C. We introduce a three-layer structure for the illumination modulation and use the following nomenclature for the modulated pulse waveforms.

- A slot has duration $T_{1}$. It represents the clock timing at which the power LEDs are driven. Hence $T_{1}$ is the resolution at which the LEDs can be modulated.

- A block has duration $T_{2}=N_{1} T_{1}$ and represents the duration of one chip. The ratio $1: N_{1}$ is the contrast ratio and also the resolution of the possible adjustment of the duty cycle for dimming purposes. This corresponds to $q=$ $\log _{2} N_{1}$ bits in dimming capabilities. In one block duration, an LED emits one pulse. The unique property of this modulation method is that the pulse could extend beyond the boundary of the block, according to the illumination requirements.

- A frame has duration $T_{3}=N_{2} T_{2}$ and it is the time interval during which one identifier is transmitted, and one measurement of the illumination intensity of each LED is made. Hence, when $T_{3}$ is in the order of a tenth of a second, requirement $\mathrm{j}$ ) is satisfied.

As can be understood from the signal description in the next sections, our modulation and multiple-access schemes attempt to satisfy requirements b) and a), in blocks and frames, respectively.

\section{A. Block Modulation Format}

Here we introduce binary PPM and PWM for a pulse of amplitude $A_{l}$ and timing reference $\tau_{l}$ with $\tau_{l} \in\left\{0,1, \ldots, N_{1}-1\right\}$. These are illustrated in Fig. 2, together with the non-modulated pulse.

1) Non-Modulated Pulse: For the non-modulated driving pulse used for dimming the LED light output, as illustrated in Fig. 2(a), we have the following:

- the starting position of all pulses for the $l$ th LED equals $\tau_{l}$;

- the pulse width in terms of number of slots is given by

$$
w_{l}=\left\lfloor p_{l} N_{1}\right\rceil
$$

where $[\cdot]$ rounds to the nearest integer and $\left(1-p_{l}\right)$ is the dimming level of the $l$ th LED, with the duty cycle $0 \leq p_{l} \leq$ 1 . Note that the dimming level is a parameter that can be different for different LEDs.

2) Pulse Position Data Modulation (PPM): For PPM, as illustrated in Fig. 2(b):
- The starting position of the $j$ th pulse for the $l$ th LED, with $j=0, \ldots, N_{2}-1$ and $l=1, \ldots, L$, is given by

$$
\delta_{j, l}= \begin{cases}\tau_{l}, & a_{j, l}=-1 \\ \tau_{l}+k_{l}, & a_{j, l}=1\end{cases}
$$

where $\tau_{l}$ and $k_{l}$ are the allocated time slot and the modulation depth for the $l$ th LED, respectively, and $a_{j, l}$ denotes the $j$ th chip of the spreading sequence or identification code for the $l$ th LED, where $a_{j, l} \in\{+1,-1\}$. The length of the spreading sequence is $\mathrm{N}_{2}$.

- The pulse width is as defined in (1).

3) Pulse Width Data Modulation (PWM): For PWM, as illustrated in Fig. 2(c):

- The starting position of the $j$ th pulse for the $l$ th LED is the same as used for PPM, i.e., it is given by $\delta_{j, l}$ as defined in (2).

- The pulse width, however, depends not only on the required light level but also on the spreading chip, i.e.

$$
w_{j, l}= \begin{cases}u_{l}, & a_{j, l}=1 \\ u_{l}+k_{l}, & a_{j, l}=-1\end{cases}
$$

where $u_{l}=\left\lfloor p_{l} N_{1}-k_{l} / 2\right\rceil$. Consequently, pulses end at $\left\{\tau_{l}+u_{l}+k_{l}\right\} \bmod N_{1}$ regardless of $a_{j, l}$. To satisfy the required duty cycle exactly over the frame interval $T_{3}$ and meet requirement a), we balance the number of 1's and -1 's in the identification/spreading code, i.e., $\sum_{j=1}^{N_{2}} a_{j, l}=$ 0 .

Evidently, if we need to embed any code, we cannot switch off the light completely. In PPM, light is emitted in at least one slot per block, so the realized duty cycle $\tilde{p}_{l} \geq 1 / N_{1}$. Similarly for PWM, we have $\tilde{p}_{l} \geq 1 /\left(2 N_{1}\right)$ for a balanced number of 1 's and -1 's in the code. If the total light level in a room must be dimmable to nonperceivable illumination levels this might present a problem. On the other hand, the minimum light level can be made arbitrarily small by choosing appropriate $T_{1}$ and $A_{l}$. Meanwhile, the maximum duty cycle also needs to remain slightly below $100 \%$, viz. $\tilde{p}_{l} \leq\left(N_{1}-1\right) / N_{1}$ and $\tilde{p}_{l} \leq\left(2 N_{1}-\right.$ 1) $/ 2 N_{1}$ for PPM and PWM, respectively. This does not lead to any practical limitation because it has only a minor impact, fractions of a percent, on the lighting intensity emitted by an LED.

\section{B. Framing}

We consider a synchronous system in which $T_{1}, N_{1}$ and $N_{2}$ are identical for all LEDs, and ideally their slots, blocks, and frames are aligned. We will omit the notational details of pulses extending into neighboring blocks, and we only consider the first frame in a continuous sequence of repetitive frames. The signal driving the $l$ th LED can be expressed as

$$
s_{l}(t)=\sum_{j=0}^{N_{2}-1} \sum_{n=0}^{N_{1}-1} s_{j, n, l} \Pi\left(\frac{t-j T_{2}-n T_{1}}{T_{1}}\right) .
$$

Here $n$ and $j$ refer to the positions of slots in a block, and to blocks in the frame, respectively, and $\Pi(t)=U(t)-U(t-1)$ is the unit pulse of unit time width and amplitude, where $U(t)$ is the unit step function. The discrete-time signal $s_{j, n, l}$ represents the sample of $s_{l}(t)$ at $t=j T_{2}+n T_{1}$. For PPM it is given by 


$$
s_{j, n, l}=A_{l} \cdot \begin{cases}0 & n=0,1, \ldots, \tau_{l}-1, \\ \frac{1-a_{j, l}}{2} & n=\tau_{l}, \ldots, \tau_{l}+k_{l}-1, \\ \frac{1+a_{j, l}}{2} & n=\tau_{l}+k_{l}, \ldots, \tau_{l}+w_{l}-1, \\ 0 & n=\tau_{l}+w_{l}, \ldots, \tau_{l}+w_{l}+k_{l}, \ldots, N_{1}-1\end{cases}
$$

while for the case of PWM, it is given by

$$
s_{j, n, l}=A_{l} \cdot \begin{cases}0 & n=0,1, \ldots, \tau_{l}-1 \\ \frac{1-a_{j, l}}{2} & n=\tau_{l}, \ldots, \tau_{l}+k_{l}-1 \\ 1 & n=\tau_{l}+k_{l}, \ldots, \tau_{l}+u_{l}+k_{l}-1 \\ 0 & n=\tau_{l}+u_{l}+k_{l}, \ldots, N_{1}-1\end{cases}
$$

These pulses can be interpreted to consist of a code-carrying prefix, a main illumination pulse and an inversely code-modulated suffix. For the PWM scheme, the duration of the suffix is reduced to zero.

\section{Multiple Access}

For our scheme, we define a two-dimensional multiple access scheme, namely in the code and time dimension. We choose CDMA to allow multiple light sources to simultaneously emit PWM-dimmed light, and nonetheless transmit unique and separable codes. We propose to use Walsh-Hadamard (WH) codes, since they can ensure perfect orthogonality and allow for computationally efficient multi-signal receiver algorithms, to address requirements e) and f), respectively. By excluding the first WH code, namely the $\{1,1, \ldots, 1\}$ DC word, all codes used have a balanced number of 1's and -1 's. This balanced property further fixes the frame-average duty cycle and shapes the illumination spectrum to make the data modulation imperceivable, to meet requirements a) and c), respectively. Moreover, the system becomes resilient to sources of constant or sufficiently slowly varying interfering light sources such as sunlight or incandescent bulbs, addressing requirement i). The $l$ th LED is assigned the identification/spreading code

$$
\mathbf{a}_{\gamma_{l}}=\left[a_{0, \gamma_{l}}, a_{1, \gamma_{l}}, \ldots, a_{N_{2}-1, \gamma_{l}}\right]^{T}
$$

which corresponds to WH code $\gamma_{l}$, with $\gamma_{l} \in\left\{2,3, \ldots, N_{2}\right\}$.

We additionally assign a timing reference $\tau_{l}$ to each LED, where each combination $\left(\tau_{l}, \gamma_{l}\right)$ is unique in the system, i.e., each combination characterizes one LED. Moreover we avoid the situation that a part of the prefix or suffix of the light pulse of one LED overlaps with the prefix or suffix of the pulse of any other LED that has the same code assigned. The resulting coded waveform over a frame is illustrated in Fig. 3.

Hence, perfectly synchronized CTDMA-PWM, meets the requirements a) and e), because the signals from LED $l$ with $\left(\tau_{l}, \gamma_{l}\right)$ and LED $l^{\prime}$ with $\left(\tau_{l^{\prime}}, \gamma_{l^{\prime}}\right)$ are orthogonal if $\left\{\tau_{l}, \tau_{l}+1, \ldots, \tau_{l}+k_{l}-1\right\} \cap\left\{\tau_{l^{\prime}}, \tau_{l^{\prime}}+1, \ldots, \tau_{l^{\prime}}+k_{l^{\prime}}-1,\right\}=\emptyset$ even if $\gamma_{l}=\gamma_{l^{\prime}}$. Consequently, if all LEDs have the same $k_{l}=k_{l^{\prime}}=k$, the upper bound to the number of LEDs that can be uniquely tagged equals $N_{1}\left(N_{2}-1\right) / k$. For CTDMA-PPM, one needs to avoid not only overlap of prefixes but also of suffixes, which can be challenging due to LED-dependent time-varying changes of the dimming levels. So PPM does not appear to be favorable from the perspective of the number of

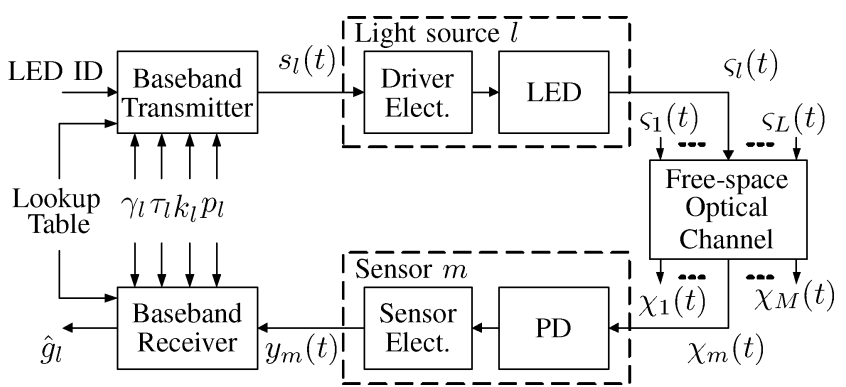

Fig. 4. Block diagram of the link between the $l$ th light source and $m$ th sensor.

LEDs that can be supported simultaneously in a convenient manner. A pragmatic approach is to let $\tau_{l}$ to be the same for every LED, while only the code $\gamma_{l}$ differs.

The proposed multiple access method allows the sensor to detect multiple signals from LEDs, simultaneously, independent of the different illumination levels, meeting requirements a) and e).

\section{Channel Model}

As shown in the system block diagram of Fig. 4, the channel is described by the relation between the signal $s_{l}(t)$ at the output of the $l$ th LED driver, and the signal $y_{m}(t)$, at the output of the $m$ th sensor. The corresponding optical transmit and receive signals are denoted in the figure as $\varsigma_{l}(t)$ and $\chi_{m}(t)$, respectively. In the following, we will consider only one sensor and thus omit the index $m$.

\section{A. Electrooptical Conversion}

The optical transmit signal $\varsigma_{l}(t)$ in response to a step function of the electrical transmit signal $s_{l}(t)=A_{l} U(t)$ is given by $\varsigma_{l}(t)=A_{l} \eta_{l} h_{\text {on }}(t)$ [22], where $\eta_{l}$ denotes the LED responsivity. The unit step response of LEDs appears approximately an exponential function [23], i.e.

$$
\begin{aligned}
h_{\text {on }}(t) & =U(t)\left[1-\exp \left(-t / \tau_{\text {on }}\right)\right] \\
h_{\text {off }}(t) & =1-U(t)\left[1-\exp \left(-t / \tau_{\text {off }}\right)\right] .
\end{aligned}
$$

The time constants $\tau_{\text {on }}$ and $\tau_{\text {off }}$ for on- and off-switching tend to differ [24]. Ignoring a possibly more complicated interaction between the on- and off-tails for very short pulses, the CTDMA-PPM optical signal becomes

$$
\begin{aligned}
\varsigma_{l}(t)=\sum_{j=0}^{N_{2}-1} A_{l} \eta_{l} h_{\mathrm{on}}\left(t-\delta_{j, l} T_{1}-j T_{2}\right) \\
\cdot h_{\mathrm{off}}\left(t-\left(\delta_{j, l}+w_{l}\right) T_{1}-j T_{2}\right) .
\end{aligned}
$$

For CTDMA-PWM, $\varsigma_{l}(t)$ is also given by (10), except that $w_{l}$ is replaced by $w_{j, l}$ given in (3).

\section{B. Indoor Light Propagation}

The LED light reaches the sensor possibly via multiple paths, as studied for instance by [6], [7], [25]-[30]. To address requirements b) and j), we will have $T_{1} \approx 1 \mu$ s for typical commercial power LEDs. For such a slot time, the intersymbol interference caused by multipath propagation can be considered negligible. Yet, reflections may change the color content of the light and, as 
a result, the detector sees different channel amplitudes for differently colored LED light. This is in agreement with requirement d). For the analyses in this paper, however, we model a line-of-sight (LOS) path. This is motivated by the fact that due to the narrow LED beams the main illumination contributions will reach the sensor via a direct LOS path.

The gain of such an optical path is

$$
\alpha_{l}=\frac{1}{r_{l}^{2}} R\left(\phi_{l}\right) \mathcal{A} \cos \left(\psi_{l}\right)
$$

where $r_{l}$ is the propagation distance, $\mathcal{A}$ is the area of the photodiode (PD) and the angle $\psi_{l}$ defines the orientation of LED $l$. Further, $R\left(\phi_{l}\right)$ describes the LED radiation pattern, which we assume to be rotationally symmetric and includes the effects of the lens. The angle $\phi_{l}$ defines the orientation of the PD with respect to the $l$ th LED. We can model $R\left(\phi_{l}\right)$ by a generalized Lambertian law [6], [22]

$$
R\left(\phi_{l}\right)=\frac{\mu+1}{2 \pi} \cos ^{\mu}\left(\phi_{l}\right)
$$

where $\mu$ represents the Lambertian mode number.

\section{Optoelectrical Conversion}

The PD in the receiver converts the incoming optical signal into the electrical signal $y(t)$. Its responsivity is denoted by $\varepsilon_{l}$, which is color dependent and thus carries index $l$. The response speed of PDs is generally much higher than that of power LEDs, hence, the switching effects can be considered negligible [22], [31].

Here we assume a receiver with a bandwidth of $1 / T_{1}$, with a flat frequency response in the signal band. In a practical system, however, one might consider to apply AC-coupling to remove background light and to reduce the required dynamic range of the receiver. This dynamic range is very dependent on the number of light sources contributing to the local light effect and will consequently change from scenario to scenario.

\section{Channel Disturbances}

There are three major sources of channel disturbances, i.e., the background light, the electronics noise and the PD shot noise.

The background light consists of light contributions from other ambient sources, which in the envisaged LED lighting system would be mostly sunlight, if at all. Its power is denoted by $\zeta(t)$. Fluctuations of $\zeta(t)$ that are slower than $T_{3}^{-1}$ can be approximated as DC during each frame, which is valid for sunlight and, hence, the argument $t$ is omitted. It is moreover assumed that the background light does not result in saturation of the receiver.

Electronics noise is mostly created by the transimpedance amplifier for the PD signal, and predominantly behaves as additive Gaussian (thermal) noise. For a power spectral density (PSD) $S_{\text {th }}\left[\mathrm{A}^{2} / \mathrm{Hz}\right]$ in an effective bandwidth $B_{\mathrm{n}}$, the variance of the thermal noise equals

$$
\sigma_{\mathrm{th}}^{2}=S_{\mathrm{th}} B_{\mathrm{n}} .
$$

Shot noise is due to a stream of electrons that are generated at random times in the PD. It is approximately Gaussian distributed due to the central limit theorem [7]. The shot noise power $\sigma_{\text {shot }}^{2}$ is linearly proportional to all light shed on the PD surface, not only including the light from LEDs, but also the background light. Hence it can be expressed as

$$
\sigma_{\text {shot }}^{2}(t)=2 q_{\mathrm{e}} B_{\mathrm{n}}\left(\sum_{l=1}^{L} \alpha_{l} \varepsilon_{l} \varsigma_{l}(t)+\varepsilon_{\zeta} \zeta\right)
$$

where $q_{\mathrm{e}}$ is the electrical charge of an electron $\left(q_{\mathrm{e}}=1.6\right.$. $10^{-19}$ Coulomb), $L$ denotes the total number of LEDs and $\varepsilon_{\zeta}$ is the PD responsivity to background light. Due to the definition of the LED signals $s_{l}(t)$ in (4), within a frame the shot noise is approximately cyclostationary with period $T_{2}$, i.e., $\sigma_{\text {shot }}^{2}(t) \approx$ $\sigma_{\text {shot }}^{2}\left(t+j T_{2}\right) \triangleq \sigma_{\text {shot }, j}^{2}(t)$ for $j=1, \ldots, N_{2}-1$.

Hence, the received electrical signal can be written as

$$
y(t)=\sum_{l=1}^{L} \alpha_{l} \varepsilon_{l} \varsigma_{l}(t)+v_{\zeta}+v_{\text {th }}(t)+v_{\text {shot }}(t)
$$

where $v_{\zeta}=\varepsilon_{\zeta} \zeta, v_{\text {th }}(t)$ and $v_{\text {shot }}(t)$ denote the background light contribution, thermal and shot noise terms, respectively. The variances for the latter two are defined in (13) and (14).

\section{Receiver Design and Performance Analysis}

The baseband receiver processing consists of three main steps. To achieve a low-complexity receiver implementation, we first apply integrate-and-dump (I\&D) processing to the received signal to yield a discrete time signal. This is followed by CDMA despreading with the assigned CDMA codes to obtain the despreaded signal values for each $\tau$ and code in the second step. In the third step we apply an estimator to find the illumination intensities for the different light sources. In this paper we focus on linear estimators, due to requirement $f$ ).

The I\&D receiver outputs the $N_{2} \times N_{1}$ matrix $\mathbf{Y}$, whose $(j, n)$ th element $y_{j, n}=\left(1 / T_{1}\right) \int_{j T_{2}+(n-1) T_{1}}^{j T_{2}+n T_{1}} y(t) \mathrm{d} t$. Hence, $\mathbf{Y}$ captures an entire frame. For the purpose of CDMA despreading with respect to the $l$ th LED, we use a submatrix of $\mathbf{Y}$, denoted by $\mathbf{Y}_{l}$, that contains the columns with the data measurements related to the $l$ th LED. For CTDMA-PPM these are, by design, the columns $n=\tau_{l}, \ldots, \tau_{l}+2 k_{l}-1$ and $n=$ $\tau_{l}+w_{l}, \ldots, \tau_{l}+w_{l}+2 k_{l}-1$ of $\mathbf{Y}$, relating to the data carrying pre- and suffix, respectively. For CTDMA-PWM the columns $n=\tau_{l}, \ldots, \tau_{l}+2 k_{l}-1$ of $\mathbf{Y}$ form $\mathbf{Y}_{l}$.

Specifically, for CTDMA-PWM, the $(j, i)$ th element of $\mathbf{Y}_{l}$ can be written as

$$
\begin{aligned}
\mathbf{Y}_{l}[j, i]= & g_{l}\left[\frac{1-a_{j, l}}{2} H_{\mathrm{on}, l}(i, i+1)\right. \\
& \left.+\frac{1+a_{j, l}}{2} H_{\mathrm{on}, l}\left(i-k_{l}, i+1-k_{l}\right)\right] \\
& +\sum_{\tilde{l}_{:} \tau_{\tilde{l}}=\tau_{l}, \gamma_{\imath} \neq \gamma_{l}} g_{\tilde{l}}\left[\frac{1-a_{j, \tilde{l}}}{2} H_{\mathrm{on}, \tilde{l}}(i, i+1)+\frac{1+a_{j, \tilde{l}}}{2}\right. \\
& \left.\quad \times H_{\mathrm{on}, \tilde{l}}\left(i-k_{\tilde{l}}, i+1-k_{\tilde{l}}\right)\right] \\
& +C_{\mathrm{dc}}+v_{j, i}
\end{aligned}
$$

where $H_{\mathrm{on}, l}\left(n_{1}, n_{2}\right) \triangleq\left(\varepsilon_{l} / T_{1}\right) \int_{n_{1} T_{1}}^{n_{2} T_{1}} h_{\mathrm{on}}(t) \mathrm{d} t$. The variable $g_{l}=A_{l} \alpha_{l} \eta_{l}$ is the individual light intensity from the $l$ th LED at the sensor location and the parameter to be estimated. The DC bias $C_{\mathrm{dc}}$ is due to the illumination pulses from other LEDs and 
the background light. The noise term $v_{j, i}$ is zero-mean Gaussian distributed with variance $\sigma_{\text {th }}^{2}+\sigma_{\text {shot }}^{2}$. The elements of $\mathbf{Y}_{l}$ for CTDMA-PPM can be obtained similarly.

The despreaded signal vector $\mathbf{d}_{l}$ is then found by multiplication of the matrix $\mathbf{Y}_{l}$ with the code vector $\mathbf{a}_{\gamma_{l}}$, as defined in (7). The result is given by the vector

$$
\mathbf{d}_{l}=\frac{2}{N_{2}} \mathbf{Y}_{l}^{T} \mathbf{a}_{\gamma_{l}}
$$

of size $4 k_{l} \times 1$ and $2 k_{l} \times 1$ for CTDMA-PPM and CTDMAPWM, respectively.

Let $\mathbf{d}_{\text {noise }}$ denote a zero-mean Gaussian distributed noise vector, modelling both the thermal and shot noises. The variance of the $p$ th element equals $\sigma_{p}^{2}=4\left(\sigma_{\text {th }}^{2}+\sigma_{\text {shot, } p}^{2}\right) / N_{2}$. Also, let us define the vector $\mathbf{h}_{l}$, which, if $\tau_{\text {on }}$ and $\tau_{\text {off }}$ are small with respect to $w_{l}$ and $k_{l}$, equals $\left[\mathbf{H}_{l, \text { on }}, \mathbf{H}_{l \text {,off }}\right]$ and $\mathbf{H}_{l \text {,on }}$ for CTDMA-PPM and CTDMA-PWM, respectively. Here the $2 k_{l} \times 1$ vectors $\mathbf{H}_{l, \text { on }}$ and $\mathbf{H}_{l, \text { off }}$ are given by

$$
\begin{aligned}
& \mathbf{H}_{l, \mathrm{on}}=\left[H_{\mathrm{on}, l}(0,1), \cdots, H_{\mathrm{on}, l}\left(k_{l}-1, k_{l}\right)\right. \text {, } \\
& H_{\mathrm{on}, l}\left(k_{l}, k_{l}+1\right)-H_{\mathrm{on}, l}(0,1), \cdots \\
& \left.H_{\mathrm{on}, l}\left(2 k_{l}-1,2 k_{l}\right)-H_{\mathrm{on}, l}\left(k_{l}-1, k_{l}\right)\right]^{T} \\
& \mathbf{H}_{l, \mathrm{on}}=\left[H_{\mathrm{off}, l}(0,1), \cdots, H_{\mathrm{off}, l}\left(k_{l}-1, k_{l}\right)\right. \text {, } \\
& H_{\text {off }, l}\left(k_{l}, k_{l}+1\right)-H_{\text {off }, l}(0,1), \cdots \\
& \left.H_{\text {off }, l}\left(2 k_{l}-1,2 k_{l}\right)-H_{\text {off }, l}\left(k_{l}-1, k_{l}\right)\right]^{T}
\end{aligned}
$$

respectively. We note that, since $\tau_{\text {on }}$ and $\tau_{\text {off }}$ are static characteristics of the LED light sources used in the system, it is reasonable to assume that the receiver has knowledge about these and thus knows $\mathbf{h}_{l}$. The parameters $\tau_{\text {on }}$ and $\tau_{\text {off }}$ can be characterized during installation of the system or derived from data sheets of the LEDs.

Using these definitions we can rewrite the despreaded signal vector, as defined in (17), to

$$
\mathbf{d}_{l}=\frac{2}{N_{2}} \mathbf{Y}_{l}^{T} \mathbf{a}_{\gamma_{l}}=g_{l} \mathbf{h}_{l}+\mathbf{d}_{\text {noise }}
$$

The illumination pulses and background light $v_{\zeta}$, together denoted as $C_{\mathrm{dc}}$, can be considered as modulated light applying the WH-code with $\gamma_{l}=1$, i.e., the dc code, which is orthogonal to all codes applied in the pre- and suffixes. Hence, the illumination pulses and background light are perfectly removed by the despreading of (17). Hence, requirement i) is satisfied.

For perfectly synchronized systems, the multiuser interference (MUI) between LEDs is zero in (20), since orthogonal codes are assigned to the LEDs applying the same slots for data modulation. Requirement e) is thus satisfied. In practical systems, however, some level of timing inaccuracies might be experienced, the influence of which is considered in Section VI.

One can use our receiver structure to obtain a real-time estimate of the shot noise variance $\sigma_{\text {shot }}^{2}$. This value can be used to design a bit detection and intensity estimation algorithms in the following sections. For that we correlate the received signal matrix for the $l$ th LED $Y_{l}$ with the dc code, yielding a vector containing the sum of the background light and illumination pulses, denoted by $\mathbf{d}_{l, \mathrm{DC}}$. From (14), $\mathbf{d}_{l, \mathrm{DC}}$ is related to the shot noise variance.

\section{A. Intensity Estimation}

It was explained in Section II that it is essential to measure the individual light level $g_{l}$ in an intelligent light system. A linear estimator of $g_{l}$ can be written as a multiplication of the despreaded vector $\mathbf{d}_{l}$ with the weighting vector $\xi_{l}$ of size $2 k_{l} \times 1$ and $4 k_{l} \times 1$ for CTDMA-PWM and CTDMA-PPM, respectively. The result is given by

$$
\hat{g}_{l}=\boldsymbol{\xi}_{l}^{T} \mathbf{d}_{l}
$$

One can choose the weight vector $\xi_{l}$ according to the least squares (LS) and the minimum mean square error (MMSE) criteria, which reflect the cases that the variance of $\mathbf{d}_{\text {noise }}$ is unknown and known, respectively.

1) LS Estimation: For LS estimation of $g_{l}$, the weight vector becomes

$$
\boldsymbol{\xi}_{l, \mathrm{LS}}=\left(\mathbf{h}_{l}^{T} \mathbf{h}_{l}\right)^{-1} \mathbf{h}_{l}
$$

For the special case in which power LEDs are switched on and off instantaneously, i.e., $\tau_{\text {on }}, \tau_{\text {off }} \ll T_{1}$, and that the elements of $\mathbf{d}_{\text {noise }}$ are i.i.d., $\boldsymbol{\xi}_{l}$ can be chosen according to equal gain combining. Hence, the $p$ th element of $\boldsymbol{\xi}_{l}$, denoted by $\xi_{l, p}$, equals 1 for $0 \leq p \leq k_{l}-1$ (for both CTDMA-PPM and CTDMAPWM) and for $2 k_{l} \leq p \leq 3 k_{l}-1$ (for CTDMA-PPM) and $\xi_{l, p}=0$, otherwise.

For performance analysis, we write $\hat{g}_{l}$ obtained through the LS estimation as

$$
\hat{g}_{l, \mathrm{LS}}=g_{l}+\left(\mathbf{h}_{l}^{T} \mathbf{h}_{l}\right)^{-1} \mathbf{h}_{l}^{T} \mathbf{d}_{\text {noise }} .
$$

The second term is the estimation error, which is a zero-mean random Gaussian variable with mean squared value

$$
\sigma_{\mathrm{LS}, l}^{2}=\left(\mathbf{h}_{l}^{T} \mathbf{h}_{l}\right)^{-2} \mathbf{h}_{l}^{T} \mathbf{R}_{\text {noise }} \mathbf{h}_{l}
$$

where $\mathbf{R}_{\text {noise }}=\mathbb{E}\left[\mathbf{d}_{\text {noise }} \mathbf{d}_{\text {noise }}^{T}\right]$ and $\mathbb{E}$ denotes expectation. If the noise is i.i.d, i.e. $\mathbf{R}_{\text {noise }}=\sigma^{2} \mathbf{I}$, where $\mathbf{I}$ is the identity matrix, then

$$
\sigma_{\mathrm{LS}, l}^{2}=\frac{\sigma^{2}}{\left\|\mathbf{h}_{l}\right\|^{2}}=\frac{4\left(\sigma_{\mathrm{th}}^{2}+\sigma_{\mathrm{shot}}^{2}\right)}{N_{2}\left\|\mathbf{h}_{l}\right\|^{2}}
$$

where $\|\cdot\|$ denotes the Euclidean norm. For this scenario, we can calculate the lower bound on the mean squared error (MSE). It can be easily shown that this is achieved when $\tau_{\text {on }}=\tau_{\text {off }}=0$ and is given by

$$
\begin{aligned}
\sigma_{\mathrm{LS}, l, \mathrm{PPM}}^{2} & \geq \frac{2\left(\sigma_{\mathrm{th}}^{2}+\sigma_{\text {shot }}^{2}\right)}{k_{l} N_{2} \varepsilon_{l}^{2}} \\
\sigma_{\mathrm{LS}, l, \mathrm{PWM}}^{2} & \geq \frac{4\left(\sigma_{\mathrm{th}}^{2}+\sigma_{\text {shot }}^{2}\right)}{k_{l} N_{2} \varepsilon_{l}^{2}}
\end{aligned}
$$

for CTDMA-PPM and CTDMA-PWM, respectively.

It can be observed that the MSE for CTDMA-PPM is half of that for CTDMA-PWM. The LS estimation process suffers from a reduction of SNR due to the nonideal response of the LEDs, i.e., the MSE will increase with increasing $\tau_{\text {on }}$ and $\tau_{\text {off }}$. Moreover, an increase in code length $N_{2}$ and modulation depth $k_{l}$ increases the estimation performance. 
2) Linear MMSE Estimation: The LS intensity estimator is unbiased, but it suffers from noise enhancement. Therefore we explore the use of the MMSE estimator. MMSE estimation for known $\mathbf{R}_{\text {noise }}$ can be realized by estimating the variance of the shot noise through the DC-code contribution, as explained at the beginning of this section.

The MMSE estimator is found as the estimator that minimizes the cost function

$$
\begin{aligned}
\sigma_{l, \mathrm{MMSE}}^{2} & =\mathbb{E}\left[\left(\hat{g}_{l}-g_{l}\right)^{2}\right]=\mathbb{E}\left[\left(\boldsymbol{\xi}_{l}^{T} \mathbf{d}_{l}-g_{l}\right)^{2}\right] \\
& =g_{l}^{2}-2 \mathbb{E}\left[g_{i} \boldsymbol{\xi}_{l}^{T} \mathbf{d}_{l}\right]+\mathbb{E}\left[\boldsymbol{\xi}_{l}^{T} \mathbf{d}_{l} \mathbf{d}_{l}^{T} \boldsymbol{\xi}_{l}\right] .
\end{aligned}
$$

Consequently, the MMSE estimator can be shown to be given by

$$
\boldsymbol{\xi}_{l, \mathrm{MMSE}}=\left(1 / \mathbb{E}\left[g_{l}^{2}\right]+\mathbf{h}_{l}^{T} \mathbf{R}_{\text {noise }}^{-1} \mathbf{h}_{l}\right)^{-1} \mathbf{R}_{\text {noise }}^{-1} \mathbf{h}_{l}
$$

and the resulting mean squared estimation error equals

$$
\sigma_{\mathrm{MMSE}, l}^{2}=\left(1 / \mathbb{E}\left[g_{l}^{2}\right]+\mathbf{h}_{l}^{T} \mathbf{R}_{\text {noise }}^{-1} \mathbf{h}_{l}\right)^{-1} .
$$

For i.i.d. noise, $\sigma_{\mathrm{MMSE}, l}^{2}$ is given by

$$
\sigma_{\mathrm{MMSE}, l}^{2}=\frac{\sigma^{2}}{\frac{\sigma^{2}}{\mathbb{E}\left[g_{l}^{2}\right]}+\left\|\mathbf{h}_{l}\right\|^{2}}=\frac{\left(\sigma_{\mathrm{th}}^{2}+\sigma_{\mathrm{shot}}^{2}\right)}{\frac{\left(\sigma_{\mathrm{th}}^{2}+\sigma_{\text {shot }}^{2}\right)}{\mathbb{E}\left[g_{l}^{2}\right]}+\frac{N_{2}\left\|\mathbf{h}_{l}\right\|^{2}}{4}} .
$$

Similarly as for the LS estimator, the lower bound of $\sigma_{\mathrm{MMSE}, l}^{2}$ can be obtained as

$$
\begin{gathered}
\sigma_{\mathrm{MMSE}, l, \mathrm{PPM}}^{2} \geq \frac{\sigma_{\mathrm{th}}^{2}+\sigma_{\mathrm{shot}}^{2}}{\frac{\sigma_{\mathrm{th}}^{2}+\sigma_{\mathrm{shot}}^{2}}{\mathbb{E}\left[g_{l}^{2}\right]}+\frac{k_{l} N_{2} \varepsilon_{l}^{2}}{2}} \\
\sigma_{\mathrm{MMSE}, l, \mathrm{PWM}}^{2} \geq \frac{\sigma_{\mathrm{th}}^{2}+\sigma_{\mathrm{shot}}^{2}}{\frac{\sigma_{\mathrm{th}}^{2}+\sigma_{\mathrm{shot}}^{2}}{\mathbb{E}\left[g_{l}^{2}\right]}+\frac{k_{l} N_{2} \varepsilon_{l}^{2}}{4}}
\end{gathered}
$$

for CTDMA-PPM and CTDMA-PWM, respectively.

It can be observed from (26), (27), (32), and (33) that when the SNR is high, the MMSE estimator and LS estimator tend to become equivalent. The performance difference between CTDMA-PPM and CTDMA-PWM is similar as for the LS estimator.

Numerical results for the intensity estimation performance will be given in Section VII-A.

\section{B. Complexity Evaluation}

In this subsection we evaluate the computational complexity required in the receiver and intensity estimation.

In every frame period, i.e., $T_{3}$, the receiver writes $N_{1} N_{2}$ samples to its memory to form $\mathbf{Y}$. It is then most efficient to implement the correlation processing of (17) for all LEDs simultaneously. This is done by applying $N_{1} \mathrm{WH}$ transforms to $\mathbf{Y}$. Each transform involves $N_{2} \log \left(N_{2}\right)$ real additions.

Let $N_{\mathrm{c}}$ denote the number of samples combined for each intensity estimation in (21). Hence, $N_{\mathrm{c}}$ equals the number of non-zero elements in $\boldsymbol{\xi}_{l}$, which for PWM is smaller than $2 k_{l}$ and for PPM is smaller than $4 k_{l}$. Each LS intensity estimation requires $N_{\mathrm{c}}$ multiplications, $N_{\mathrm{c}}-1$ additions and one abs $(\cdot)$ operation. Each MMSE intensity estimation, requires, however, inverting a $N_{\mathrm{c}} \times N_{\mathrm{c}}$ matrix, in addition to the computations needed for LS intensity estimation.

Let us consider a basic implementation using $k_{l}=1 \forall l$ and CTDMA-PWM, where the switching speed of LEDs are very high, i.e., $\tau_{\text {on }} \ll T_{1}$ and $\tau_{\text {off }} \ll T_{1}$. Each LS intensity estimate requires one $\operatorname{abs}(\cdot)$ operation and each MMSE estimation requires one addition, one division and one $\operatorname{abs}(\cdot)$ operation. Hence, the complexity of such detector is low, addressing requirement $\mathrm{f}$ ).

This simple receiver can make an estimate of the light intensity from $\left(N_{2}-1\right)$ LEDs applying an orthogonal code for each time division bin of $k$ slots wide. Hence the average number of estimates per slot of length $T_{1}$ becomes

$$
S_{1}=\frac{\left(N_{2}-1\right) N_{1}}{k} \frac{1}{N_{1} N_{2}}=\frac{\left(N_{2}-1\right)}{N_{2}} \frac{1}{k} .
$$

For large $N_{2}$ and $k=1$ this tends to unity. Hence the system can make close to one LED intensity measurement per $T_{1}$. We believe that thereby we reach a fundamental limit for binary (on-off) modulation. Meanwhile, the schemes allow an arbitrary dimming of each LED independently, at a resolution of $2^{q}=N_{1}$ steps to meet requirement b). Although the LEDs are clocked at a rate of $T_{1}^{-1}$, in each block, i.e., $T_{2}$, only a single power-on and power-off transition is made. This limits the capacitive losses to address requirement $h$ ).

\section{IMPACT OF TIMING INACCURACIES}

In the previous sections, we considered perfectly synchronized LED light sources. In a practical lighting system, however, certain timing inaccuracy between the different light sources and between the light sources and the sensor will occur, e.g., due to cabling and imperfections in local clocks. One can distinguish two influences: a fixed timing offset and timing jitter. In this section we will consider these two effects to be independent. In other words, when considering the timing jitter, we assume that the fixed offset for every LED is zero. In this way, we can obtain clear insights on the performance impact of these two kinds of timing inaccuracies, separately. Let us define $\varphi_{l}$ and $\tilde{\varphi}_{l}$ to denote the fixed offset and random jitter between the signals from the $l$ th LED and that of the receiver, respectively. Further, the variance of $\tilde{\varphi}_{l}$ is denoted by $\sigma_{\varphi_{l}}^{2}$.

Fig. 5 illustrates possible pulse waveforms from different LEDs in the presence of timing inaccuracies. These inaccuracies will potentially lead to errors in the matrix $\mathbf{Y}_{l}$ for all $l$, and consequently in the decision variable $\mathbf{d}_{l}$. This in turn will result in an error in the estimation of the illumination contribution $g_{l}$. This error will be evaluated in this section, in which we will consider the CTDMA-PWM scheme and for simplicity of explanation we consider the case of $\left\{\tau_{\text {on }}, \tau_{\text {off }}\right\} \ll T_{1}$.

Specifically, in the presence of $\varphi_{l}$, we now get that (16) is changed into (35), shown at the bottom of the next page, where the definition on the group $\left\{l^{\prime}\right\}$ and $\left\{l^{\prime \prime}\right\}$ is given below. Further, the DC term $\tilde{C}_{\mathrm{dc}}$ also varies due to the timing error of the LEDs in the group $\left\{l^{\prime \prime \prime}\right\}$ and $\left\{l^{\prime \prime \prime \prime}\right\}$, as also introduced later.

In terms of the resulting errors in $\mathbf{d}_{l}$ due to the fixed offset and jitter, we need to consider four potential types of errors, as illustrated in Fig. 5.

1) The first error is in the vector $\mathbf{h}_{l}$ of the considered $l$ th LED, as defined in Section V. The resulting vector is written as 


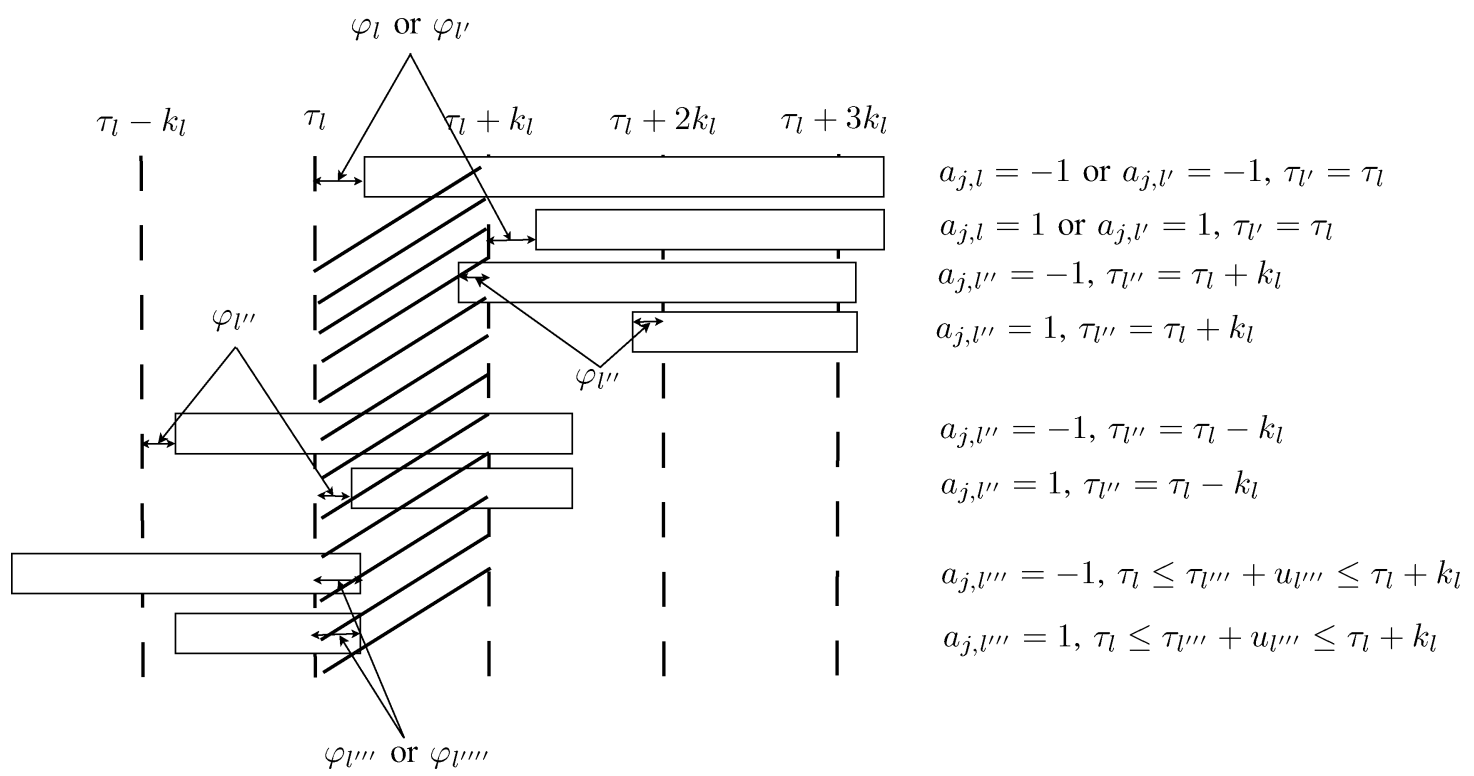

Fig. 5. Examples of each of the four types of MUI due to timing errors for different LEDs. The shaded area is the period that the detector gathers signal for the $l$ th LED.

$\tilde{\mathbf{h}}_{l}$. The influence of this error is observed in the first term of (35).

2) The second error is the interference from the set of LEDs, denoted by $\left\{l^{\prime}\right\}$, that are assigned the same time slot as that of the $l$ th LED, but have different WH codes. That is, the LEDs for which $\tau_{l^{\prime}}=\tau_{l}$ and $\gamma_{l^{\prime}} \neq \gamma_{l}$.

3) The third potential type of error is the interference from the set of LEDs in neighboring slots, denoted by $\left\{l^{\prime \prime}\right\}$, i.e., $\tau_{l^{\prime \prime}}=\tau_{l}+k_{l}$ or $\tau_{l^{\prime \prime}}=\tau_{l}-k_{l^{\prime \prime}}$. Although under perfect synchronization the signals from the $l^{\prime \prime}$ th and $l$ th LED are decoupled in the time domain, these can possibly be mixed together under timing errors. We only need to consider the LED that are assigned the neighboring slots, since the timing offsets are relatively small, i.e., $\left|\varphi_{l}\right| \ll T_{1}$ and $\left|\sigma_{\varphi_{l}}\right| \ll T_{1}$.

4) The fourth potential type of error is the interference from two sets of LEDs, denoted by $\left\{l^{\prime \prime \prime}\right\}$ and $\left\{l^{\prime \prime \prime \prime}\right\}$, for which the tails of their illumination pulses lie in the slots where the light of the lth LED is modulated by the code. That is, the LEDs for which $\tau_{l^{\prime \prime \prime}}+u_{l^{\prime \prime \prime}}=\tau_{l}$ or $\tau_{l}+k_{l}$, and $\tau_{l}<\tau_{l^{\prime \prime \prime \prime}}+u_{l^{\prime \prime \prime \prime}}<\tau_{l}+k_{l}$. We only need to consider the negative values of $\varphi_{l^{\prime \prime \prime}}$ and $\tilde{\varphi}_{l^{\prime \prime \prime}}$ for $\tau_{l^{\prime \prime \prime}}+u_{l^{\prime \prime \prime}}=\tau_{l}$ and positive values for $\tau_{l^{\prime \prime \prime}}+u_{l^{\prime \prime \prime}}=\tau_{l}+k_{l}$. In contrast, we need to consider interference both if $\varphi_{l^{\prime \prime \prime \prime}}$ and $\tilde{\varphi}_{l^{\prime \prime \prime \prime}}$ are positive or negative.

\section{A. Fixed Timing Offset}

The estimation error due to fixed timing offsets is caused by error type 1 and 2. It is derived in Appendix I for the LS estimator. There it is found that the worst case error is given by

$$
\left|\hat{g}_{l}-g_{l}\right|_{\text {worst }}=\frac{\left|\varphi_{l}\right|}{k_{l} T_{1}} g_{l}+\sum_{l^{\prime \prime}: \gamma_{l^{\prime \prime}}=\gamma_{l}} \frac{\left|\varphi_{l^{\prime \prime}}\right|}{k_{l} T_{1}} g_{l^{\prime \prime}}+v
$$

where the variance of the noise term $v$ equals $4\left(\sigma_{\mathrm{th}}^{2}+\right.$ $\left.\sigma_{\text {shot }}^{2}\right) /\left(\varepsilon_{l}^{2} N_{2} k_{l}\right)$.

\section{B. Timing Jitter}

The estimation error due to timing jitter is caused by all four error types. This is elaborated in Appendix II, where we show that the error can be modelled as a Gaussian random variable. Specifically, we have that the error is given by

$$
\left|g_{l}-\hat{g}_{l}\right|=\frac{g_{l}}{k_{l} T_{1}} \sqrt{\frac{2 \sigma_{\varphi}^{2}}{\pi}}+\sum_{l^{\prime \prime}: \gamma_{l^{\prime \prime}}=\gamma_{l}} \frac{g_{l^{\prime \prime}}}{k_{l} T_{1}} \sqrt{\frac{\sigma_{\varphi}^{2}}{2 \pi}}+\tilde{v}
$$

$$
\begin{aligned}
\mathbf{Y}_{l}[j, i]= & g_{l}\left[\frac{1-a_{j, l}}{2} H_{\mathrm{on}, l}\left(i+\frac{\varphi_{l}}{T_{1}}, i+1+\frac{\varphi_{l}}{T_{1}}\right)+\frac{1+a_{j, l}}{2} H_{\mathrm{on}, l}\left(i-k_{l}+\frac{\varphi_{l}}{T_{1}}, i+1-k_{l}+\frac{\varphi_{l}}{T_{1}}\right)\right] \\
& +\sum_{l^{\prime}} g_{l^{\prime}}\left[\frac{1-a_{j, l^{\prime}}}{2} H_{\mathrm{on}, l^{\prime}}\left(i+\frac{\varphi_{l^{\prime}}}{T_{1}}, i+1+\frac{\varphi_{l^{\prime}}}{T_{1}}\right)+\frac{1+a_{j, l^{\prime}}}{2} H_{\mathrm{on}, l^{\prime}}\left(i-k_{l^{\prime}}+\frac{\varphi_{l^{\prime}}}{T_{1}}, i+1-k_{l^{\prime}}+\frac{\varphi_{l^{\prime}}}{T_{1}}\right)\right] \\
+ & \sum_{l^{\prime \prime}} g_{l^{\prime \prime}}\left[\frac{1-a_{j, l^{\prime \prime}}}{2} H_{\mathrm{on}, l^{\prime \prime}}\left(i+\tau_{l}-\tau_{l^{\prime \prime}}+\frac{\varphi_{l^{\prime \prime}}}{T_{1}}, i+1+\tau_{l}-\tau_{l^{\prime \prime}}+\frac{\varphi_{l^{\prime \prime}}}{T_{1}}\right)\right. \\
& \left.+\frac{1+a_{j, l^{\prime \prime}}}{2} H_{\mathrm{on}, l^{\prime \prime}}\left(i-k_{l^{\prime \prime}}+\tau_{l}-\tau_{l^{\prime \prime}}+\frac{\varphi_{l^{\prime \prime}}}{T_{1}}, i+1-k_{l^{\prime \prime}}+\tau_{l}-\tau_{l^{\prime \prime}}+\frac{\varphi_{l^{\prime \prime}}}{T_{1}}\right)\right]+\tilde{C}_{\mathrm{dc}}+v_{j, i}
\end{aligned}
$$


where the variance of the noise-and-interference term $\tilde{v}$ equals

$$
\begin{gathered}
\sigma_{\tilde{v}}^{2}=\frac{4\left(\sigma_{\mathrm{th}}^{2}+\sigma_{\mathrm{shot}}^{2}\right)}{\varepsilon_{l}^{2} N_{2} k_{l}}+\frac{\sigma_{\varphi}^{2}}{N_{2} k_{l}^{2} T_{1}^{2}}\left(1-\frac{1}{\pi}\right) \\
\times\left(2 \sum_{l} g_{l}^{2}+2 \sum_{l^{\prime}} g_{l^{\prime}}^{2}+\sum_{l^{\prime \prime}} g_{l^{\prime \prime}}^{2}\right. \\
\left.+2 \sum_{l^{\prime \prime \prime}} g_{l^{\prime \prime \prime}}^{2}+4 \sum_{l^{\prime \prime \prime \prime}} g_{l^{\prime \prime \prime \prime}}^{2}\right) .
\end{gathered}
$$

It can be concluded from (37) and (38) that jitter causes interference from many more LEDs than the fixed offset in (36). It is noted, however, that their influence in the variance is normalized by $1 / N_{2}$ due to the despreading, reducing the influence.

Numerical results for the influence of both the fixed timing offset and timing jitter are presented in Section VII-B.

\section{NUMERICAL RESULTS}

In this section, we present the numerical results for a large indoor environment of $20 \mathrm{~m} \times 20 \mathrm{~m}$ with a high density of LEDs. In this scenario, the LEDs are distributed in a square grid with a dimension of $25 \mathrm{~cm}$, i.e., a density of $16 \mathrm{LED}$ lamps $/ \mathrm{m}^{2}$ that all consist of a co-located red, green and blue LED. Consequently, the full ceiling is covered with a total of $3 \times 6400$ LEDs. These lamps generate light with a color temperature of $6500 \mathrm{~K}$ (D65) [32] for equal duty cycles of the red, green and blue LEDs, i.e., for $p_{\mathrm{r}}=p_{\mathrm{g}}=p_{\mathrm{b}}$. The sensor is located in the middle of the room and is oriented such that it faces the ceiling at a height of $h=3.5 \mathrm{~m}$. For the lamp and sensor parameters we consider Philips Lumileds LUXEON K2 LEDs [33] and a Hamamatsu S6468 monochromatic photodetector [34]. The Lambertian mode number $\mu$ is the same for all LEDs. The area of the PD $\mathcal{A}=100 \mathrm{~mm}^{2}$. For the red, green and blue LEDs, the driving currents $I$ are $0.25,1.1$, and 0.22 A, respectively, which corresponds to a lumen output of 43.7, 142, and 9.72. The responsivity of the LEDs $\eta$ equals $1.30,0.243$ and $0.89 \mathrm{~W} / \mathrm{A}$ and that of the $\mathrm{PD} \varepsilon$ equals $0.42,0.28,0.21 \mathrm{~A} / \mathrm{W}$ for the red, green and blue LEDs, respectively.

Walsh-Hadamard codes with $N_{2}=256$ are used, and $N_{1}$ is set to be 1024 to provide a dimming range of 10 bits and a fundamental frequency of approximately $1 \mathrm{kHz}$, which is far above the visible frequency range. Every LED takes $k_{l}=k=5$ slots for modulation, such that the spreading gain is $k N_{2}=$ 1280 , to create sufficient noise robustness. The slot period $T_{1}=$ $1 \mu$ s for which we can assume $\tau_{\text {on }} \ll T_{1}$ and $\tau_{\text {off }} \ll T_{1}$, since typical LEDs have response times of smaller than $100 \mathrm{~ns}$ [5]. In this simulation, all LEDs are considered to operate at the same duty cycle of $50 \%$, i.e., $p_{l}=0.5 \forall l$. The PSD of electronics noise $S_{\mathrm{th}}=1.69 \times 10^{-24} \mathrm{~A}^{2} / \mathrm{Hz}$. While all the LEDs are radiating light and embedding codes simultaneously, we consider only the sensing performances of the link between one LED and the sensor. Slots and codes are randomly assigned to the different LEDs.

In this scenario with high LED density, shot noise is dominated by the light contributions of the LEDs, i.e., the first term in (14). This large room scenario can be considered a worst case scenario, since many LEDs contribute to the shot noise.

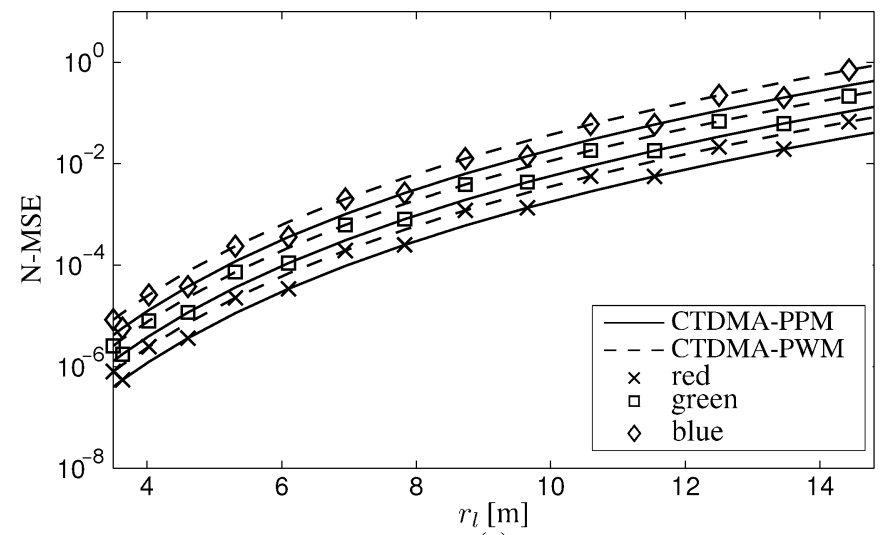

(a)

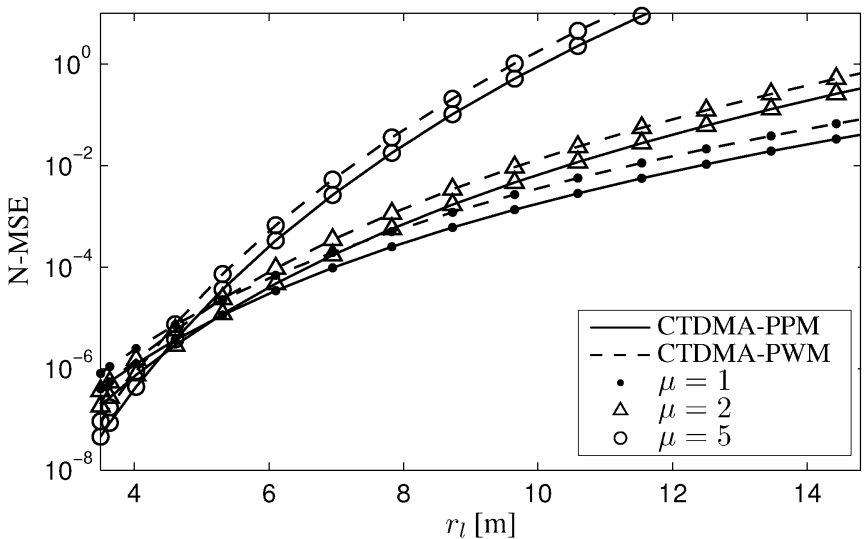

(b)

Fig. 6. Normalized MSE in intensity estimation versus PD-LED hypotenuse propagation distance, for a ceiling height of $h=3.5 \mathrm{~m}$. (a) Different color LEDs, $\mu=1$. (b) Red LEDs, different $\mu$ values.

\section{A. MSE in Intensity Estimation}

First we illustrate the MSE performance in LS intensity estimation, as derived analytically in (25), for the large room scenario. To this end we study in Fig. 6 the normalized MSE (N-MSE) in light intensity estimation, i.e., the MSE results derived in Section V-A normalized by the squared value of the actual light intensity. We present the MSE results as a function of $r_{l}$, the hypotenuse propagation distance according to $r_{l}=\sqrt{h^{2}+x_{l}^{2}+y_{l}^{2}}$ with $\left(x_{l}, y_{l}\right)$ the ceiling grid coordinates of the light source with respect to the sensor. The sensor is fixed and we take numerical results for LEDs at different locations on the ceiling.

Fig. 6(a) depicts the N-MSE for the three color LEDs with $\mu=1$. It is observed from the depicted results that the performance in intensity estimation degrades in an exponential fashion as the distance between the LED and sensor increases. We can observe that we get considerable estimation errors, N-MSE > $10^{-2}$, in the intensity estimation using CTDMA-PPM for LEDs whose distances from the sensor are above $12.5,10.7$, and $9.3 \mathrm{~m}$ for red, green and blue LEDs, respectively. Over the considered range the difference in color seems to result in a shift of the MSE curves, which again can be attributed to the difference in optical output power and color dependence of the PD responsivity. For CTDMA-PWM these ranges are approximately $1 \mathrm{~m}$ smaller, as expected from the results in (26) and (27). The impact of this 
error, however, is limited since the contribution to the local illumination from LEDs which are beyond these distances are negligible, since their contributions are more than $20 \mathrm{~dB}$ lower than that of the LED with the dominant contribution at the sensing location. The above shows that we can satisfy requirement d) up to considerable ranges.

Similar results for red LEDs with different radiation patterns, for $\mu=1,2$ and 5, are depicted in Fig. 6(b). For the CTDMA-PPM scheme, N-MSE values below $10^{-2}$ are now achieved up to distances of 12.5, 10.5, and $7.6 \mathrm{~m}$ for $\mu$ equals 1,2 , and 5 , respectively. The degradation in range for CTDMA-PWM, compared to CTDMA-PPM, is less than $1 \mathrm{~m}$. In contrast to the difference in color, the difference in LED radiation pattern changes the slope of the MSE curve. For very low distances the performance is slightly improved for high $\mu$, i.e., narrow light beams, while for high distances the MSE is significantly increased for high $\mu$.

\section{B. Effect of Timing Errors}

The influence of timing errors on the estimation performance is illustrated in Fig. 7. The results are depicted in terms of N-MSE in light intensity estimation for different distances between the PD and LEDs. LEDs with $\mu=1$ are considered and the $l$ th LED, for which the N-MSE is calculated, is red.

The result for worst case error for a fixed timing offset, as derived in (36), are reported in Fig. 7(a) as function of $\varphi / T_{1}=$ $\left|\varphi_{l}\right| / T_{1}=\left|\varphi_{l^{\prime \prime}}\right| / T_{1}$. We can conclude from this figure that for low values of $\varphi$ the performance is limited by the noise. For larger values the interference becomes dominant. A N-MSE of $10^{-2}$ can be achieved for $\varphi / T_{1}$ of $0.39,0.34,0.05$, and 0.01 for a PD-LED distance $r_{l}$ of 3.5, 4.6, 7.8, and $9.7 \mathrm{~m}$, respectively. The N-MSE for $13.5 \mathrm{~m}$ is already above $10^{-2}$ without timing offset.

The timing jitter results of (37) and (38) are illustrated in Fig. 7(b). This figure shows the N-MSE as function of the normalized timing jitter variance $\sigma_{\varphi}^{2} / T_{1}^{2}$. Again, the performance is limited for low values of $\sigma_{\varphi}^{2}$ by the noise and for higher values by the jitter-caused interference. A N-MSE performance of $10^{-2}$ can be achieved for $\sigma_{\varphi}^{2} / T_{1}^{2}$ of $0.2,0.15,5 \cdot 10^{-3}$ and $7 \cdot 10^{-4}$ for a PD-LED distance $r_{l}$ of $3.5,4.6,7.8$, and $9.7 \mathrm{~m}$, respectively. The N-MSE for $13.5 \mathrm{~m}$ is above $10^{-2}$ even for zero timing jitter. Simulations for larger rooms, i.e. with more LEDs, achieved approximately the same performance, which indicates that the interference is dominated by the contributions of a few strong LEDs (near the sensor).

Hence, we can conclude that timing inaccuracies will only yield considerable errors for the estimation of illumination contributions of LEDs that are located far from the sensor. Since their light contribution at the sensor location are also small, the impact of this on the final system performance will be minor.

\section{Accuracy in Light Color Estimation}

Instead of calculating BER performance as typically occurs in (wireless) optical communication-oriented papers, in this section we illustrate the performance of the proposed methods to estimate and set the color of the light emitted by the color LED lamps, using a monochromatic sensor. In this procedure the sensor receiver performs an intensity estimation for every LED.

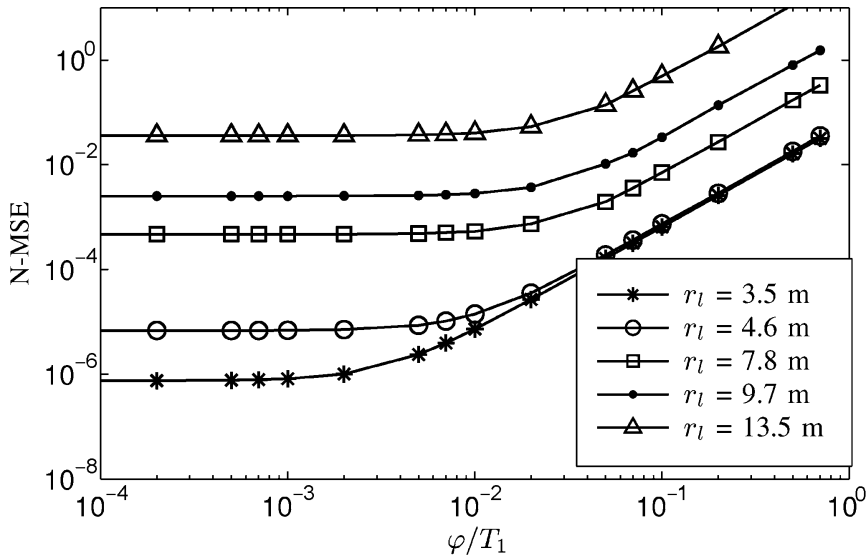

(a)

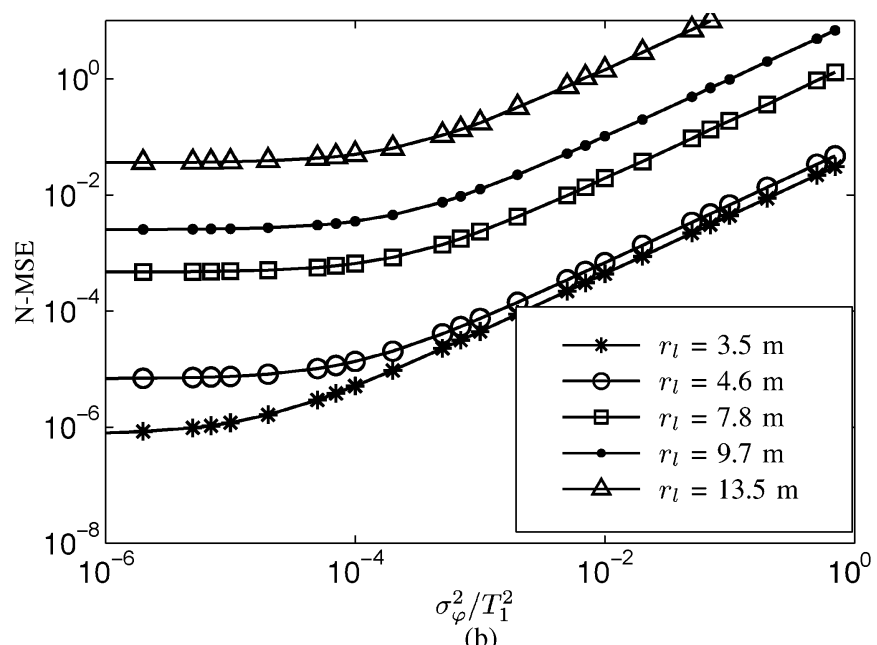

(b)

Fig. 7. Normalized MSE in intensity estimation under timing errors for different PD-LED hypotenuse propagation distances, for a ceiling height of $h=$ $3.5 \mathrm{~m}$. (a) Influence of fixed timing offset. (b) Influence of timing jitter.

Since it also identifies the LEDs, it can obtain the color of the individual LEDs from the master controller and combine this with the intensity estimates to determine the color of the resulting light. When the system can accurately estimate the color of the resulting light, these estimates are also suitable to locally render colors with a control loop.

To study the performance in estimation of local light, we use Monte Carlo simulations of the light color estimation based on the LS estimates of the intensities of the individual colored LEDs with $\mu=1$. We refer the interested reader to text books on color theory, e.g., [19] and [32], which show that the accuracy in color estimation is best evaluated in the commonly used $u^{\prime} v^{\prime}$ uniform chromaticity diagram. This diagram is normalized to the sensitivity of the human eye, such that the threshold value for a noticeable color difference is independent of the $u^{\prime} v^{\prime}$ color point of the light. A color difference is only noticeable if the error, i.e., $\Delta u^{\prime} v^{\prime}$, is larger than 0.001 [4], [19], [35].

Fig. 8(a) depicts the $u^{\prime} v^{\prime}$ points of the primary colors of the LEDs (red, green and blue) and that of 4 mixed colors. The mixed colors are set by varying the duty cycles for the different color LEDs. For the colors white (D65), pink, light green and purple, the ratios between the duty cycles of the red, green and 


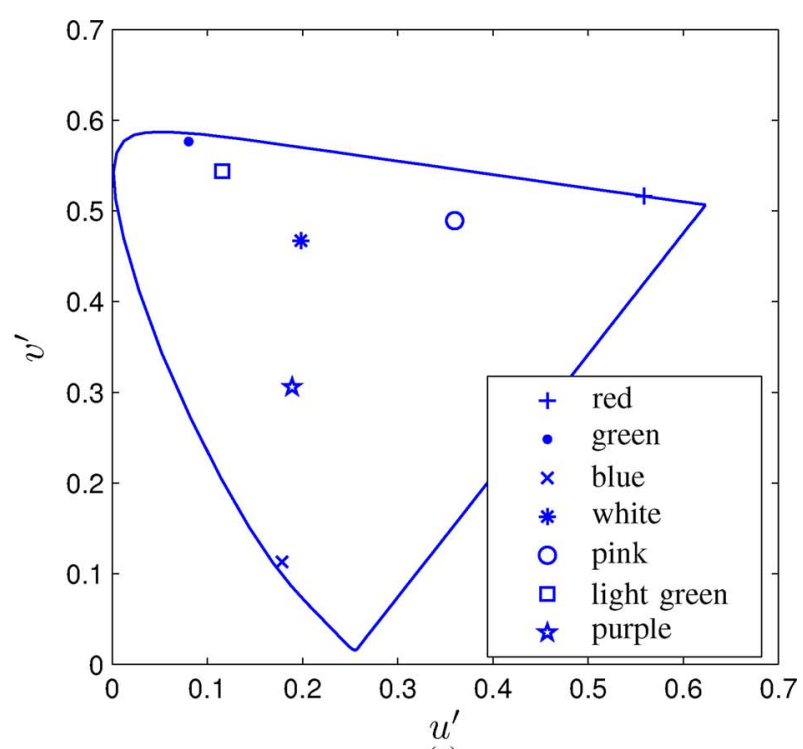

(a)

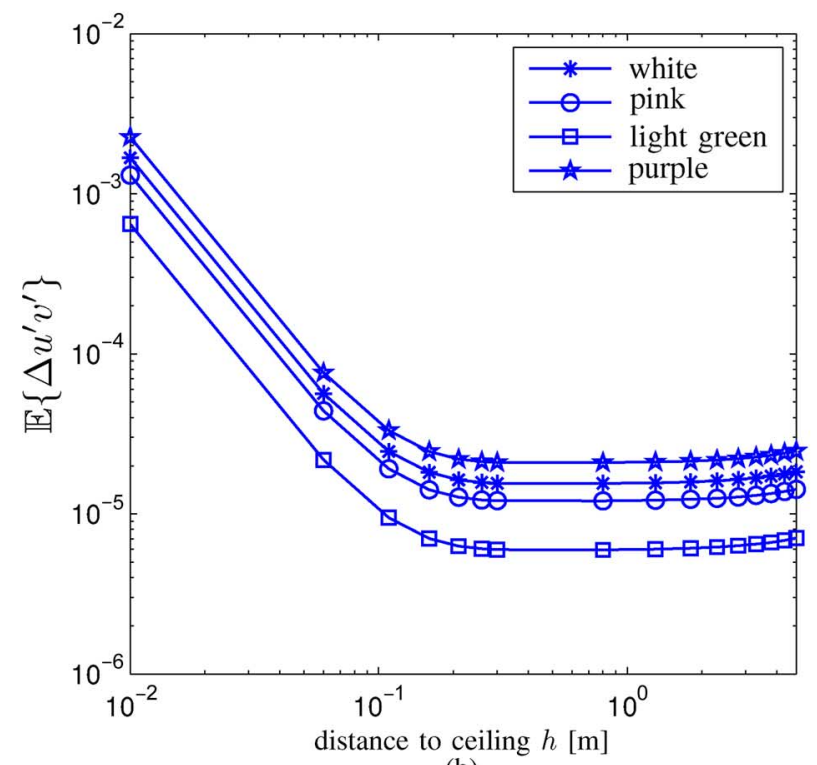

(b)

Fig. 8. Accuracy in color estimation for different mixed colors. (a) LED and mixed color in $u^{\prime} v^{\prime}$ diagram. (b) $\mathbb{E}\left\{\Delta u^{\prime} v^{\prime}\right\}$ for different mixed colors.

blue LEDs are given by 1:1:1, 1:0.2:0.2, 0.2:1:0.2, and 0.2:0.2:1, respectively. All lamps contribute the same color.

We transformed the simulated estimates (21) into estimated coordinates $\hat{u}^{\prime}$ and $\hat{v}^{\prime}$ of $u^{\prime}$ and $v^{\prime}$, respectively, [19], [32]. The mean of $\Delta u^{\prime} v^{\prime}$ is then given $\mathbb{E}\left\{\Delta u^{\prime} v^{\prime}\right\}=\mathbb{E}\left\{\sqrt{\left(\hat{v}^{\prime}-v^{\prime}\right)^{2}+\left(\hat{u}^{\prime}-u^{\prime}\right)^{2}}\right\}$. This mean is plotted in Fig. 8(b) as a function of the perpendicular distance to the ceiling $h$ for the four mixed colors. We conclude from Fig. 8(b) that inside the simulated room for all four rendered colors the mean of $\Delta u^{\prime} v^{\prime}$ stays well below the threshold of noticeable color difference. Also we observe a slight difference in color estimation performance for the different color points. This can be explained by the color dependence of the MSE in intensity estimation, as observed in Fig. 6(a). Furthermore, the dependence on height can be attributed to the location and height dependence of the MSE, where it is noted that the performance is quite stable except for sensor locations near the ceiling, in between lamps. This can be explained by the rapidly decreasing signal contributions at these height due to the downward radiation pattern of the LEDs.

The accurate estimation of the color point of the purple light appears to be the most challenging, which corresponds with the fact that the MSE in intensity estimation is highest for the blue LEDs. On the other hand, the color estimation of pink is worse than that of the light green light, although the MSE for the red LEDs is the lowest. This can be attributed to the transformation to the $u^{\prime} v^{\prime}$ plane, which takes into account the sensitivity of the human visual perception system, which is less sensitive to differences in green colors. However, the difference in $\Delta u^{\prime} v^{\prime}$ between the different colors is very small and well below the visible threshold, satisfying requirement $d$ ). So the proposed method more than adequately estimates the color point of the produced light with a monochromatic sensor.

Extensions to the studied system could be made by the use of a multicolor sensor, which would allow for all three color LEDs in one lamp to use the same $\left(\tau_{l}, \gamma_{l}\right)$.

\section{Evaluation}

For the large room scenario considered in this section, we proposed a dimming resolution of $q=10$ bits, i.e. $N_{1}=1024$. Furthermore, we applied a system with $N_{2}=256, k=5$ and $T_{1}=1 \mu \mathrm{sec}$. Thus LEDs are switched around $10^{3}$ times per second, which keeps the capacitive power losses due to on- and off-transients negligibly small, addressing requirement $h$ ), yet it is fast enough to prevent visible flickering. Each LED can be measured within a $0.26 \mathrm{~s}$ period, to address requirement $\mathrm{j}$ ), and the system can simultaneously and separately detect the light from more than 250,000 independent light sources, to satisfy requirement e).

For systems with fewer LEDs, one can decrease the measurement time by choosing a smaller code length $N_{2}$. A code length of 32, for example, would yield a measurement of the intensities of all LEDs each $33 \mathrm{~ms}$, while still more than 30,000 LEDs can be supported.

\section{DISCUSSIONS AND CONCLUSIONS}

Solid-state lighting will play an increasingly important role in general illumination as well as light effect creation. The intuitive control of sophisticated lighting installations is regarded as a key factor for the market acceptance of such systems. This paper introduced a technology named coded light that brings such simplicity by enabling new interaction modes for lighting systems. The technology is based on the embedding of identifiers in the light from high brightness LEDs. This allows for identification and estimation of the light contribution of each LED in a localized manner, which is essential to the deployment of new user interaction modes.

A set of requirements for such systems were formulated in this paper, which significantly differ from those for the usual wireless optical transmission systems studied hitherto. The differences are caused by the fact that the proposed system is used simultaneously for illumination, transmission of identifiers and lighting control. To satisfy these requirements, we introduced a new class of binary modulation methods suitable for PWM- 
based dimming, simultaneous identification of multiple SSL luminaires, and estimation of their individual local light contributions. These methods involve the use of a main illumination pulse with a data carrying prefix and/or suffix, and are based on PPM and PWM. A hybrid code and time division multiple access scheme is used to separate the signals from the different LEDs, which allows for a large number of light sources to be measured by the system simultaneously.

A receiver design was proposed. For estimation, the least squares and minimum mean square error estimators were studied. Furthermore, the performance of the intensity estimators were derived analytically. The results reveal that the CTDMA-PPM scheme is $3 \mathrm{~dB}$ more robust to noise than the CTDMA-PWM scheme in MSE performance. This, however, comes at the cost of a more complicated code and time slot assignment for CTDMA-PPM and a smaller number of LEDs that can be allocated. Moreover, we analyzed the influence of imperfect timing on the estimation performance.

For a worst-case scenario with a very large number of LEDs in a room, we evaluated numerically the main system performance parameters. Next to the MSE of RGB color components, we calculated the achievable color accuracy in the $u^{\prime} v^{\prime}$ color triangle. We concluded from this study that our approach provides the required MSE performance up to sensor-LED distances of about 7 to 10 meters. It was, moreover, concluded that the error in the estimation of the light color is very small and well below the visible threshold, even for this highly demanding scenario. A topic for future research is the numerical analysis of the system taking into account reflections.

\section{APPENDIX I}

\section{FIXED TIMING OFFSET}

In this section, we consider the case when there is a fixed timing offset, $\varphi_{l}$, between the clock of the $l$ th LED and that of the receiver. We can then investigate the four types of errors as defined in Section VI.

With respect to the first type of error, we get that the $i$ th element of the vector $\tilde{\mathbf{h}}_{l}$ equals $\tilde{\mathbf{h}}_{l, i}=H_{\mathrm{on}, l}\left(i+\varphi_{l} / T_{1}, i+1+\right.$ $\left.\varphi_{l} / T_{1}\right)-H_{\mathrm{on}, l}\left(i-k_{l}+\varphi_{l} / T_{1}, i+1-k_{l}+\varphi_{l} / T_{1}\right)$.

For the second type of error, since the timing offset of the $l^{\prime}$ th LED is fixed, the influence on the values of $\mathbf{Y}_{l}$ can be seen to be proportional to the codeword vector $\gamma_{l^{\prime}}$. Thus, during the despreading operation (20), the signals due to the $l^{\prime}$ th LED is cancelled out due to the orthogonal property of the WH codes.

Similarly, the influence on $\mathbf{Y}_{l}$ due to the $l^{\prime \prime}$ th LED is also proportional to $\gamma_{l^{\prime \prime}}$. Therefore, we only need to consider the LEDs in the set $\left\{l^{\prime \prime}\right\}$ such that $\gamma_{l^{\prime \prime}}=\gamma_{l}$. Specifically, we can get the interference on $\mathbf{d}_{l}$ due to the $l^{\prime \prime}$ th LED can be written to be $g_{l^{\prime \prime}} \mathbf{q} l^{\prime \prime}$, where the $i$ th element of $\mathbf{q} l^{\prime \prime}$ is ${ }^{1}$

$$
\begin{aligned}
\mathbf{q}_{l^{\prime \prime}, i}= & H_{\mathrm{on}, l^{\prime \prime}}\left(i+k_{l^{\prime \prime}}+\varphi_{l^{\prime \prime}} / T_{1}, i+1+2 k_{l^{\prime \prime}}+\varphi_{l^{\prime \prime}} / T_{1}\right) \\
& -H_{\mathrm{on}, l^{\prime \prime}}\left(i+k_{l^{\prime \prime}}+\varphi_{l^{\prime \prime}} / T_{1}, i+1+k_{l^{\prime \prime}}+\varphi_{l^{\prime \prime}} / T_{1}\right), \\
& \text { if } \tau_{l^{\prime \prime}}=\tau_{l}-k_{l^{\prime \prime}}
\end{aligned}
$$

${ }^{1}$ Note that the situation becomes more complicated if $\tau_{l}=0$ or $\tau_{l}=\left\lfloor N_{1} / k_{l}\right\rfloor-1$. In this case, the signal might be interfered from the LEDs with $\tau_{l^{\prime \prime}}=\left\lfloor N_{1} / k_{l}\right\rfloor-1$ or $\tau_{l^{\prime \prime}}=0$, even if $\gamma_{l^{\prime \prime}} \neq \gamma_{l}$. In practice, we can easily set certain guard slots to prevent this complication. Therefore, we do not consider these situations in this paper.

$$
\begin{aligned}
\mathbf{q}_{l^{\prime \prime}, i}= & H_{\mathrm{on}, l^{\prime \prime}}\left(i-k_{l}+\varphi_{l^{\prime \prime}} / T_{1}, i+1-k_{l}+\varphi_{l^{\prime \prime}} / T_{1}\right) \\
& -H_{\mathrm{on}, l^{\prime \prime}}\left(i-k_{l}-k_{l^{\prime \prime}}+\varphi_{l^{\prime \prime}} / T_{1},\right. \\
& \left.i+1-k_{l}-k_{l^{\prime \prime}}+\varphi_{l^{\prime \prime}} / T_{1}\right), \\
& \text { if } \tau_{l^{\prime \prime}}=\tau_{l}+k_{l} .
\end{aligned}
$$

Finally, with respect to the fourth type of error, the influence on $\mathbf{Y}_{l}$ due to the signal from the $l^{\prime \prime \prime}$ th or $l^{\prime \prime \prime \prime}$ th LED can be seen to be dc. Therefore, due to the dc-free property of the used WH codes, the signal of the $l^{\prime \prime \prime}$ th or $l^{\prime \prime \prime \prime}$ th LED will be cancelled during the WH despreading.

We can then conclude that despreaded signal vector is given by

$$
\mathbf{d}_{l}=g_{l} \tilde{\mathbf{h}}_{l}+\sum_{l^{\prime \prime}: \gamma_{l^{\prime \prime}}=\gamma_{l}} g_{l^{\prime \prime}} \mathbf{q}_{l^{\prime \prime}}+\mathbf{d}_{\text {noise }}
$$

where the influence of the timing offset is clear from comparing (41) to (20). We can then evaluate the performance in estimation of $g_{l}$. For instance, for the LS estimator we have

$$
\begin{aligned}
\hat{g}_{l}= & {\left[\mathbf{h}_{l}^{T} \mathbf{h}_{l}\right]^{-1} \mathbf{h}_{l}^{T} \mathbf{d}_{l}=g_{l}\left[\mathbf{h}_{l}^{T} \mathbf{h}_{l}\right]^{-1} \mathbf{h}_{l}^{T} \tilde{\mathbf{h}}_{l} } \\
& +\sum_{l^{\prime \prime}} g_{l^{\prime \prime}}\left[\mathbf{h}_{l}^{T} \mathbf{h}_{l}\right]^{-1} \mathbf{h}_{l}^{T} \mathbf{q}_{l^{\prime \prime}}+\left[\mathbf{h}_{l}^{T} \mathbf{h}_{l}\right]^{-1} \mathbf{h}_{l}^{T} \mathbf{d}_{\text {noise }}
\end{aligned}
$$

The sum of the errors depends on the signs of $\varphi_{l}$ and $\varphi_{l^{\prime \prime}}$. The worst case corresponds to that all errors add constructively. This worst case error is given by

$$
\begin{aligned}
\left|\hat{g}_{l}-g_{l}\right|_{\text {worst }}= & g_{l}\left|1-\left[\mathbf{h}_{l}^{T} \mathbf{h}_{l}\right]^{-1} \mathbf{h}_{l}^{T} \tilde{\mathbf{h}}_{l}\right| \\
& +\sum_{l^{\prime \prime}: \gamma_{l^{\prime \prime}}=\gamma_{l}} g_{l^{\prime \prime}}\left|\left[\mathbf{h}_{l}^{T} \mathbf{h}_{l}\right]^{-1} \mathbf{h}_{l}^{T} \mathbf{q}_{l^{\prime \prime}}\right| \\
& +\left[\mathbf{h}_{l}^{T} \mathbf{h}_{l}\right]^{-1} \mathbf{h}_{l}^{T} \mathbf{d}_{\text {noise }} \\
= & \frac{\left|\varphi_{l}\right|}{k_{l} T_{1}} g_{l}+\sum_{l^{\prime \prime}: \gamma_{l^{\prime \prime}}=\gamma_{l}} \frac{\left|\varphi_{l^{\prime \prime}}\right|}{k_{l} T_{1}} g_{l^{\prime \prime}}+v
\end{aligned}
$$

where the variance of the noise term $v$ equals $4\left(\sigma_{\text {th }}^{2}+\right.$ $\left.\sigma_{\text {shot }}^{2}\right) /\left(\varepsilon_{l}^{2} N_{2} k_{l}\right)$.

\section{APPENDIX II \\ RANDOM TIMING JITTER}

For the case of random time jitter, we assume the jitter is Gaussian distributed with variance $\sigma_{\varphi_{l}}^{2}$ for the lth LED. In the presence of the random jitter, the values $\tilde{\varphi}_{l, j}$ are independent for different chips $j$. Similar to the analysis on the fixed timing offset, we again need to consider four possible types of the estimation errors due to the random time jitter, as defined in Section VI.

First, for the $(j, i)$ th element of $\mathbf{Y}_{l}$, the output of the I\&D receiver suffers from a random bias $H_{\mathrm{on}}\left(i+\tilde{\varphi}_{l, j} / T_{1}, i+1+\right.$ $\left.\tilde{\varphi}_{l, j} / T_{1}\right)-H_{\mathrm{on}}(i, i+1)$ if $a_{l, j}=-1$ and $H_{\mathrm{on}}\left(i-k_{l}+\right.$ $\left.\tilde{\varphi}_{l, j} / T_{1}, i+1-k_{l}+\tilde{\varphi}_{l, j} / T_{1}\right)-H_{\text {on }}\left(i-k_{l}, i+1-k_{l}\right)$ if $a_{l, j}=1$. We can then compute the probability characteristics of these two terms. The probability distribution of the random bias clearly depends on the characteristics of $h_{\mathrm{on}}$. For every $j$, the interference due to the timing offset is then proportional to 
$g_{l}$ and the ratio is $\left(\tilde{\varphi}_{l, j} / T_{1}\right)$. Then, the mean and the variance of the influence on the corresponding terms of $\mathbf{Y}_{l}$ are

$$
\operatorname{mean}_{l, j}= \begin{cases}\frac{g_{l}}{T_{1} \sqrt{2 \pi \sigma_{\varphi}^{2}}} \int_{-\infty}^{0} \tilde{\varphi}_{l} e^{-\frac{\tilde{\varphi}_{l}^{2}}{2 \sigma_{\varphi}^{2}}} \mathrm{~d} \tilde{\varphi}_{l} & \\ =-\frac{g_{l}}{T_{1}} \sqrt{\frac{\sigma_{\varphi}^{2}}{2 \pi}} & \text { when } a_{l, j}=-1 \\ \frac{g_{l}}{T_{1} \sqrt{2 \pi \sigma_{\varphi}^{2}}} \int_{0}^{\infty} \tilde{\varphi}_{l} e^{-\frac{\tilde{\varphi}_{l}^{2}}{2 \sigma_{\varphi}^{2}}} \mathrm{~d} \tilde{\varphi}_{l} & \\ =\frac{g_{l}}{T_{1}} \sqrt{\frac{\sigma_{\varphi}^{2}}{2 \pi}} & \text { when } a_{l, j}=1\end{cases}
$$

$$
\begin{aligned}
\operatorname{var}_{l, j} & =\frac{g_{l}^{2}}{T_{1}^{2} \sqrt{2 \pi \sigma_{\varphi}^{2}}} \int_{-\infty}^{0} \tilde{\varphi}_{l}^{2} e^{-\frac{\varphi_{l}^{2}}{2 \sigma_{\varphi}^{2}}} \mathrm{~d} \tilde{\varphi}_{l}-\operatorname{mean}_{l, j}^{2} \\
& =g_{l}^{2} \frac{\sigma_{\varphi}^{2}}{2 T_{1}^{2}}\left(1-\frac{1}{\pi}\right)
\end{aligned}
$$

respectively. Consequently, during the despreading operation (20), we get that the influence on $\mathbf{d}_{l}$ is a random variable with mean $\left(g_{l} /\left(k_{l} T_{1}\right)\right) \sqrt{2 \sigma_{\varphi}^{2} / \pi}$ and variance $\left(2 g_{l}^{2} \sigma_{\varphi}^{2} /\left(N_{2} k_{l}^{2} T_{1}^{2}\right)\right)(1-1 / \pi)$. We note that only one of the $k_{l}$ slots is affected, and, hence, these expressions are normalized over $k_{l}$.

Second, we consider the interference from the LEDs in set $\left\{l^{\prime}\right\}$. The mean and variance of such interference for the $j$ th chip can be obtained similarly as mean $l^{\prime}, j=\left(g_{l^{\prime}} / T_{1}\right) \sqrt{\sigma_{\varphi}^{2} /(2 \pi)}$ and $\operatorname{var}_{l^{\prime}, j}=\left(g_{l^{\prime}}^{2} / T_{1}^{2}\right)\left(\sigma_{\varphi}^{2} / 2\right)(1-1 / \pi)$, respectively. Due to the orthogonality property of the WH codes, the mean will be cancelled during the despreading. Thus, there will be a random interference with zero mean and variance $\left(2 g_{l^{\prime}}^{2} \sigma_{\varphi}^{2} /\left(N_{2} k_{l}^{2} T_{1}^{2}\right)\right)(1-1 / \pi)$.

Third, the interferences from the LEDs in the set $\left\{l^{\prime \prime}\right\}$ are considered. We only need to consider the $j$ th chips with $a_{l^{\prime \prime}, j}=$ -1 , and the interference only occurs on the slot $i=\tau_{l}+$ $k_{l}-1$. The mean and variance can be obtained as $\operatorname{mean}_{l^{\prime \prime}, j}=$ $\left(g_{l^{\prime \prime}} / T_{1}\right) \sqrt{\sigma_{\varphi}^{2} /(2 \pi)}$ and $\operatorname{var}_{l^{\prime \prime}, j}=\left(g_{l^{\prime \prime}}^{2} / T_{1}^{2}\right)\left(\sigma_{\varphi}^{2} / 2\right)(1-1 / \pi)$, respectively. Similarly, we can also obtain the results for $\tau_{l^{\prime \prime}}=$ $\tau_{l}-k_{l^{\prime \prime}}$. The mean values of the interferences for $\gamma_{l^{\prime \prime}} \neq \gamma_{l}$ will be cancelled during the despreading, however there is a non-zero mean for the interference from the LED with $\gamma_{l^{\prime \prime}}=\gamma_{l}$. Therefore, in $\mathbf{d}_{l}$, the interference for each $l^{\prime \prime}$ can be characterized by a random variable with variance $\left(g_{l^{\prime \prime}}^{2} \sigma_{\varphi}^{2} /\left(N_{2} k_{l}^{2} T_{1}^{2}\right)\right)(1-1 / \pi)$ and mean $\left(g_{l^{\prime \prime}} /\left(k_{l} T_{1}\right)\right) \sqrt{\sigma_{\varphi}^{2} /(2 \pi)}$ for $\gamma_{l^{\prime \prime}} \neq \gamma_{l}$ and zero for $\gamma_{l^{\prime \prime}}=\gamma_{l}$.

Finally, we consider the interference due to the LEDs in the sets $\left\{l^{\prime \prime \prime}\right\}$ and $\left\{l^{\prime \prime \prime \prime}\right\}$. The tails of the illumination pulses from these LEDs lie in the slots between $\tau_{l}$ and $\tau_{l}+k_{l}$. For the $l^{\prime \prime \prime}$ th LED whose tail ends, ideally, at $\tau_{l} T_{1}$, the influence on $\mathbf{Y}_{l}$ at each $j$ is similar to that from the $l$ th LED itself when $a_{l, j}=1$. So, we can compute the mean and variance as mean $_{l^{\prime \prime \prime}, j}=$ $\left(g_{l^{\prime \prime \prime}} / T_{1}\right) \sqrt{\sigma_{\varphi}^{2} /(2 \pi)}$ and $\operatorname{var}_{l^{\prime \prime \prime}, j}=\left(g_{l^{\prime \prime \prime}}^{2} / T_{1}\right)\left(\sigma_{\varphi}^{2} / 2\right)(1-1 / \pi)$, respectively. The same results hold for $\tau_{l^{\prime \prime \prime}}+u_{l^{\prime \prime \prime}}=\tau_{l}+u_{l}$, except that the mean is $\operatorname{mean}_{l^{\prime \prime \prime}, j}=-\left(g_{l^{\prime \prime \prime}} / T_{1}\right) \sqrt{\sigma_{\varphi}^{2} /(2 \pi)}$. Then, after the despreading operation, we obtain the interference on $\mathbf{d}_{l}$ due to the LEDs in $\left\{l^{\prime \prime \prime}\right\}$ is characterized by a vari- ance $\left(2 g_{l^{\prime \prime \prime}}^{2} \sigma_{\varphi}^{2} /\left(N_{2} k_{l}^{2} T_{1}^{2}\right)\right)(1-1 / \pi)$. For the LEDs in the set $\left\{l^{\prime \prime \prime \prime}\right\}$, the tail of the illumination pulse end at a slot between $\tau_{l}$ and $\tau_{l}+k_{l}$. In this case, we need to consider the interference of the tails on both neighboring slots. We can compute the mean and variance of interference on each slot similarly as above. Further, after the despreading, the mean values of these interferences are always cancelled in $\mathbf{d}_{l}$. Thus, we only need to consider the variance of the interference in $\mathbf{d}_{l}$, which is $\left(4 g_{l^{\prime \prime \prime \prime}}^{2} \sigma_{\varphi}^{2} /\left(N_{2} k_{l}^{2} T_{1}^{2}\right)\right)(1-1 / \pi)$.

In summary, after despreading, due to the large number of interfering sources and the central limit theorem, the total interference can be characterized by a Gaussian random variable. The mean is determined by error types 1 and 3 . The variance is determined by all four error types.

\section{ACKNOWLEDGMENT}

The authors are grateful to Prof. J. Bergmans, Prof. S. Arnon, Dr. S. Baggen, and Dr. J. Talstra for the valuable discussions. They also thank the anonymous reviewers for their helpful comments.

\section{REFERENCES}

[1] J.-P. M. G. Linnartz, L. Feri, H. Yang, S. B. Colak, and T. C. W. Schenk, "Communications and sensing of illumination contributions in a power LED lighting system," in Proc. Int. Conf. Commun., Beijing, China, May 2008, pp. 5396-5400.

[2] E. F. Schubert and J. K. Kim, "Solid-state light sources getting smart," Science, vol. 308, pp. 1274-1278, May 2005.

[3] D. A. Steigerwald, J. C. Bhat, D. Collins, R. M. Fletcher, M. O. Holcomb, M. J. Ludowis, P. S. Martin, and S. L. Rudaz, "Illumination with solid state lighting technology," IEEE J. Sel. Topics Quantum Electron., vol. 8, pp. 310-320, Mar./Apr. 2002.

[4] S. Muthu, F. J. P. Schuurmans, and M. D. Pashley, "Red, green, and blue LEDs for white light illumination," IEEE J. Sel. Topics Quantum Electron., vol. 8, no. 2, pp. 333-338, Mar./Apr. 2002.

[5] Luxeon Star LEDs [Online]. Available: http://www.luxeonstar.com/faqs.php

[6] F. R. Gfeller and U. Bapst, "Wireless in-house communication via diffuse infrared radiation," Proc. IEEE, vol. 67, no. 11, pp. 1474-1486, Nov. 1979

[7] J. M. Kahn and J. R. Barry, "Wireless infrared communications," Proc. IEEE, vol. 85, no. 2, pp. 265-298, Feb. 1997.

[8] R. Otte, L. P. de Jong, and A. H. M. van Roermund, Low-Power Wireless Infrared Communications. New York: Springer, 1999.

[9] S. Hranilovic, Wireless Optical Communication Systems. New York: Springer, 2004.

[10] J. M. H. Elmirghani and R. A. Cryan, "New PPM-CDMA hybrid for indoor diffuse infrared channels," Electron. Lett., vol. 30, pp. 1646-1647, Sep. 1994.

[11] U. N. Griner and S. Arnon, "Multiuser diffuse indoor wireless infrared communications using equalized synchronous CDMA," IEEE Trans. Commun., vol. 54, pp. 1654-1662, Sep. 2006.

[12] G. Pang, T. Kwan, H. Liu, and C.-H. Chan, "LED wireless," IEEE Ind Appl. Mag., vol. 8, no. 1, pp. 21-28, 2002.

[13] T. Komine and M. Nakagawa, "Fundamental analysis for visible-light communication system using LED lights," IEEE Trans. Consumer Electron., vol. 50, no. 1, pp. 100-107, Feb. 2004.

[14] M. Z. Afgani, H. Haas, H. Elgala, and D. Knipp, "Visible light communication using OFDM," in Proc. TRIDENTCOM 2006, March 2003.

[15] J. Grubor, S. C. J. Lee, K.-D. Langer, A. M. J. Koonen, and J. Walewski, "Wireless high-speed data transmission with phosphorescent whitelight LEDs," in Proc. ECOC 2007, Sep. 2007.

[16] S. Miyauchi, T. Komine, S. Haruyama, and M. Nakagawa, "Analysis of LED-allocation algorithm for high-speed parallel wireless optical communication system," in Proc. RWS'06, Jan. 2006, pp. 191-194.

[17] H. Sugiyama, S. Haruyama, and M. Nakagawa, "Brightness control methods for illumination and visible-light communication systems," in Proc. ICWMC'07, Mar. 2007.

[18] J. E. Farrell, "An analytical method for predicting perceived flicker," Behav. Inf. Technol., vol. 5, pp. 349-358, 1986. 
[19] G. Wyszecki and W. S. Stiles, Color Science; Concepts and Methods, Quantitative Data and Formulas. New York: Wiley, 1967.

[20] P. R. Boyce, Human Factors in Lighting, 2nd ed. London, U.K.: Taylor and Francis, 2003.

[21] W.-H. Lan, "Wavelength shift of gallium nitride light emitting diode with p-down structure," IEEE Trans. Electron Devices, vol. 52, pp. $1217-1219,2005$

[22] D. Wood, Optoelectronic Semiconductor Devices. Englewood Cliffs, NJ: Prentice-Hall, 1994, ch. 1,2,3,5,6.

[23] A. Descombes and W. Guggenbuhl, "Investigation of the influence of several diode parameters on the light-delay time in large-area SH-junction LED's," IEEE Trans. Electron Devices, vol. ED-25, no. 3, pp. 379-382, Mar. 1978.

[24] A. Descombes and W. Guggenbuhl, "Large signal circuit model for LED's used in optical communication," IEEE Trans. Electron Devices, vol. ED-28, no. 4, pp. 395-404, Apr. 1981.

[25] C. R. Lomba, R. T. Valadas, and A. M. de O. Duarte, "Experimental characterisation and modelling of the reflection of infrared signals and indoor surfaces," Inst. Elect. Eng. Proc.-Optoelectron., vol. 145, no. 3, pp. 191-197, Jun. 1998.

[26] V. Jungnickel, V. Pohl, S. Nönnig, and C. V. Helmolt, "A physical model of the wireless infrared communication channel," IEEE J. Sel. Areas Commun., vol. 20, no. 3, pp. 631-640, Apr. 2002.

[27] J. B. Carruthers, S. M. Carroll, and P. Kannan, "Propagation modelling for indoor optical wireless communications using fast multireceiver channel estimation," Inst. Elect. Eng. Proc.-Optoelectron., vol. 150, no. 5, pp. 473-481, Oct. 2003.

[28] Y. A. Alqudah and M. Kavehrad, "MIMO characterization of indoor wireless optical link using a diffuse-transmission configuration," IEEE Trans. Commun., vol. 51, no. 9, pp. 1554-1560, Sep. 2003.

[29] M. R. Pakravan, M. Kavehrad, and H. Hashemi, "Indoor wireless infrared channel characterization by measurements," IEEE Trans. Veh. Technol., vol. 50, no. 4, pp. 1053-1073, Jul. 2001.

[30] O. Gonzalez, S. Rodriguez, R. Perez-Jimenez, B. R. Mendoza, and A. Ayala, "Error analysis of the simulated impulse reponse on indoor wireless optical channels using a Monte Carlo-based ray-tracing algorithm," IEEE Trans. Commun., vol. 53, no. 1, pp. 124-130, Jan. 2005.

[31] A. E. Iverson and D. L. Smith, "Mathematical modeling of photoconductor transient response," IEEE Trans. Electron Devices, vol. 34, pp. 2098-2107, Oct. 1987.

[32] R. Hunt, The Reproduction of Color, 6th ed. New York: Wiley, 2004.

[33] Philips Lumileds, Tech. Datasheet DS51, Power Light Source LUXEON K2 [Online]. Available: http://www.lumileds.com/ 2007

[34] Hamamatsu, Product Sheet S6468, High-Speed Sensor With Preamp [Online]. Available: http://www.hamamatsu.com/ Jan. 2007

[35] P. Rizzo, A. Bierman, and M. S. Rea, "Color and brightness discrimination of white LEDs," in Proc. SPIE, Solid State Lighting II, 2002, vol. 4776 , pp. $235-246$.

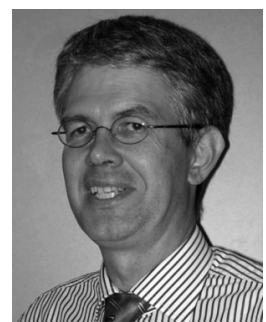

Jean-Paul M. G. Linnartz (S'85-M'87-SM'99) received the M.Sc. degree (cum laude) from Eindhoven University of Technology (TU/e), Eindhoven, The Netherlands, in 1986, and the Ph.D. degree (cum laude) from Delft University of Technology (TU Delft), The Netherlands, in December 1991.

He is a Senior Technology Director with Philips Research, Eindhoven, where he is responsible for research in the domain of sensor networks and systems. Previously, he headed security and connectivity research groups. He is also a part-time professor with TU/e. He joined Philips in 1995, initially to set up a research activity on digital rights management for multimedia content and security systems. He applied signal detection principles in the field of electronic watermarking, and invented various attacks and security measures. He introduced a privacy-preserving method of biometric identification that prevents misuse of templates from data bases. He proposed algorithms to mitigate Doppler intercarrier interference in OFDM reception for NXP chip sets for mobile DVB television reception. He introduced coded light, a modulation scheme that allows the embedding of identifiers in light sources, to facilitate convenient control of illumination. He holds 25 patents. During 1992-1995, he was an Assistant Professor with the University of California at Berkeley. In 1993, he proposed and analyzed multicarrier CDMA. From 1988 to 1991 and in 1994, he was an Assistant and Associate Professor with TU Delft, respectively.

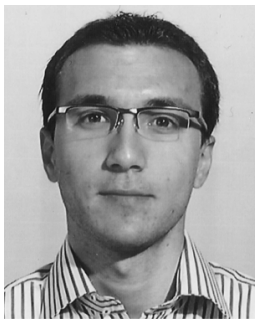

Lorenzo Feri (M'07) received the M.Sc. degree in telecommunication engineering from University of Pisa, Italy, in 2004. As part of his master program, he spent seven months at Delft University of Technology (TU Delft), The Netherlands, to perform research on Ultra Wide Band.

Since 2005, he has been with the Distributed Sensor Systems Group, Philips Research Laboratories, Eindhoven, The Netherlands, as a Research Scientist and project leader. His research interest lies and applied signal processing. in visible light communications, lighting controls,

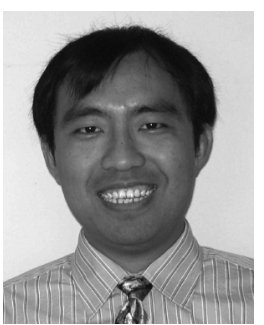

Honging Yang (S'06) received the B.S. and M.S. degrees from the Department of Electronic Engineering, Tsinghua University, Beijing, China, in 2000 and 2003 , respectively. He also received the M.E. degree from the Department of Electrical and Computer Engineering, National University of Singapore, Singapore, in 2005.

$\mathrm{He}$ is now working toward the Ph.D. degree with Eindhoven University of Technology (TU/e), Eindhoven, The Netherlands, while conducting joint research with Philips Research Laboratories, Eindhoven. His research interest lies in signal processing for illumination systems, digital communications, and recording systems.

Sel B. Colak received the $\mathrm{Ph} . \mathrm{D}$. degree in quantum electronics from the University of California at Los Angeles, in 1978.

Since his graduation, he has been with Philips Research Laboratories, Eindhoven, The Netherlands, as a Principal Scientist and/or Project Leader, first at Briarcliff Manor, NY, then in Eindhoven. His work has been primarily on computational and theoretical modeling of various devices and physical phenomena. This includes topics such as laser light scattering, semiconductor electronic FET devices in Si and Si-Ge, rare-Earth and transition-metal ion spectroscopy and lasers, band-offsets and electronic states in GaAs quantum-well structures, GaAs and $\mathrm{ZnSe}$ semiconductor injection lasers and electron beampumped lasers, photoluminescence and electronic transport in II-VI semiconductors, second-harmonic generation from GaAs laser light in KTP waveguides, electronic and optoelectronic neural network hardware, image reconstruction for medical optical tomography, and most recently, optical wireless communications. He has contributed to many of the experimental works in these areas. $\mathrm{He}$ is the author of more than 50 publications and holds over 15 patents on these topics.

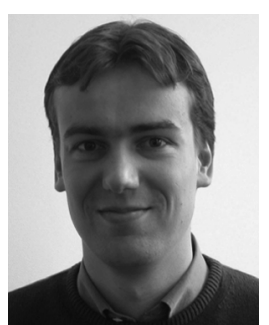

Tim C. W. Schenk (S'01-M'07) received the M.Sc. and Ph.D. degrees in electrical engineering from Eindhoven University of Technology (TU/e), Eindhoven, The Netherlands, in 2002 and 2006, respectively.

From 2002 to 2004, he was with the Wireless Systems Research Group, Agere Systems, Nieuwegein, The Netherlands. From 2004 to 2006, he was a Research Assistant with the Radiocommunications Group, TU/e. Since 2006, he is with Philips Research Laboratories, Eindhoven, as a Senior Scientist and Cluster Leader in the Distributed Sensor Systems department. His current research focuses on sensor networks, applied signal processing, and networked control systems. He authored the book RF Imperfections in High-Rate Wireless Systems: Impact and Digital Compensation (The Netherlands: Springer, 2008), and numerous scientific publications in the field of signal processing and communications. He is inventor of over 30 patents and patent applications.

Dr. Schenk was awarded the 2006 Veder Award from the Dutch Scientific Radio Fund Veder for his contributions in the field of system optimization for multiple-antenna systems. 